\title{
8. DIAGENETIC TRENDS IN LEG 126 SANDSTONES ${ }^{1}$
}

\author{
Kathleen M. Marsaglia ${ }^{2}$ and $\mathrm{Kazue} \mathrm{Tazaki}^{3}$
}

\begin{abstract}
Early diagenesis in Leg 126 forearc and backarc sands/sandstones is characterized by the dissolution of intermediate to mafic brown glass, the alteration of colorless rhyolitic glass to clay minerals, precipitation of thin clay-mineral rim cements, and minor precipitation of clinoptilolite cements. Later, more intense diagenesis is restricted to Oligocene forearc basin sediments at Sites 787, 792, and 793. In these sections, the effects of early diagenesis have been intensified and overprinted by later diagenetic effects including (1) large-scale dissolution of feldspar and pyroxene crystals, (2) further dissolution of vitric components, (3) precipitation of minor carbonate cements, and (4) pervasive, multiple-staged zeolite cementation. Zeolite minerals present include analcite, mordenite, natrolite, heulandite, wairakite, chabazite, erionite, herschelite, and phillipsite. The latest diagenetic events appear to be the minor dissolution of zeolite cements and the precipitation of minor carbonate and potassium feldspar(?) cements. Observed porosity types include primary interparticles; primary intraparticles in vesicular glass and foraminifers; primary interparticles reduced by compaction and cementation; secondary intraparticles produced by dissolution of feldspar, nonopaque heavy minerals, volcanic glass, and foraminifer tests; and secondary interparticles produced by the dissolution of zeolite cements. Within forearc Oligocene sections at Sites 787 and 792, diagenetic effects appear to decrease with depth in the Oligocene section; however, at Site 793 the majority of samples are intensely altered.
\end{abstract}

\section{INTRODUCTION}

Holes drilled on Ocean Drilling Program (ODP) Leg 126 completely penetrated the sedimentary fill and recovered basement rocks at sites within the Sumisu Rift, an incipient backarc basin, and the Izu-Bonin forearc basin (see Figs. 1, 2, and 3 in Marsaglia, this volume). Two sites were drilled within the Sumisu Rift (Sites 790 and 791), one successful site on the rift flank (Site 788), and three sites within the forearc basin (Sites 787, 792, and 793). Pore waters in the Sumisu Rift (Sites 790 and 791) were surprisingly unaltered, with little evidence for hydrothermal activity expected in an actively spreading intra-arc rift (Taylor, Fujioka, et al., 1990). In contrast, pore waters recovered at Sites 792 and 793 in the forearc basin section were extremely altered. The $\mathrm{CaCl}_{2}$ brines within the forearc sediments were the most extensively altered pore waters ever analyzed in the history of the Deep Sea Drilling Project/Ocean Drilling Program (Egeberg et al., 1990; Taylor, Fujioka, et al., 1990). The composition of these pore waters has been attributed to diagenetic reactions within the volcaniclastic basin fill, most notably alteration of volcanogenic materials resulting in the neoformation of zeolites and clay minerals (smectite/chlorite) (Egeberg et al., 1990; Taylor, Fujioka, et al., 1990).

The purposes of this study are to document the lithification processes and diagenetic trends within Leg 126 volcaniclastic sandstones and to compare and contrast early vs. late diagenetic styles across the Izu-Bonin arc system. The findings presented herein are preliminary in nature and set the stage for later detailed investigations.

\section{METHODS}

Lithified and semilithified volcaniclastic sandstones from Sites 787 , 788, 790, 791, 792, and 793 were sampled for this study. Rock chips were cut perpendicular to bedding $\left(30 \mathrm{~cm}^{3}\right.$ samples were divided into

\footnotetext{
${ }^{1}$ Taylor, B., Fujioka, K., et al., 1992. Proc. ODP, Sci. Results, 126: College Station, TX (Ocean Drilling Program).

Department of Geological Sciences, The University of Texas at El Paso, El Paso, TX 79968, U.S.A.

${ }^{3}$ Department of Geology, Shimane University, Matsue, Shimane Prefecture 690 ,
Japan.
}

three portions shared by K. Fujioka [Fujioka and Saito, this volume] and R. Hiscott [Hiscott and Gill, this volurne]). A small portion of each sample (approximately $1.0-0.5 \mathrm{~cm}^{3}$ broken off the end of a $10-\mathrm{cm}^{3} \mathrm{chip}$ ) was retained for chemical analysis, and then the remaining rock chip was high-pressure, vacuum-impregnated with bluedyed epoxy to aid in the porosity studies. Thin sections prepared from the impregnated chips were uniformly stained for both potassiumand calcium-bearing feldspars. Because this staining technique is a critical portion of this study, it is outlined in the Appendix. Each thin section was then examined by means of standard petrographic techniques using a Nikon Labophot-pol research microscope at The University of Texas at El Paso. Reserved portions of selected samples were analyzed by means of X-ray powder diffraction (XRD) techniques and a scanning electron microscope (SEM) equipped with an energy dispersive X-ray analyzer (EDX) at Shimane University, Japan.

\section{ANALYTIC RESULTS}

Analytic results for 20 samples from Sites 787, 788, 792, and 793 are summarized in Table 1, and X-ray patterns for all but the sample from Site 788 are displayed in Figures 1, 2, and 3. The authigenic minerals identified in these samples include smectite and a wide variety of zeolites. Zeolite minerals identified include analcite, mordenite, natrolite, heulandite, wairakite, chabazite, erionite, herschelite, and phillipsite. These minerals can also be identified by their characteristic morphologies and elemental composition using scanning electron microscopy (Plates 1 and 2). The Site 787, 788, and 792 samples are characterized by the presence of analcite, wairakite, and phillipsite whereas Site 793 samples contain a wider variety of zeolite minerals: analcite, mordenite, natrolite, heulandite, wairakite, chabazite, erionite, herschelite, and phillipsite.

Analcite, clinoptilolite, and phillipsite are common components of deep-sea sediments, whereas the other zeolite minerals present in Leg 126 sandstones are not commonly found in deep-sea sediments (Kastner, 1981). Wairakite is primarily associated with hydrothermal deposits (Hay, 1977; Boles, 1977); the estimated minimum temperature of wairakite formation ranges from $75^{\circ} \mathrm{C}$ (Seki et al., 1969) to $200^{\circ} \mathrm{C}$ (Coombs et al., 1959). 
Table 1. Summary of X-ray diffraction results for Leg 126 sandstones.

\begin{tabular}{|c|c|c|}
\hline $\begin{array}{l}\text { Core, section, } \\
\text { interval (cm) }\end{array}$ & $\begin{array}{l}\text { Depth } \\
\text { (mbsf) }\end{array}$ & Mineralogy \\
\hline $\begin{array}{l}\text { 126-787B- } \\
15 R-1,108-110 \\
15 R-4,26-29 \\
17 R-5,92-95 \\
18 R-2,42-44 \\
31 R-1,135-138 \\
32 R-1,54-56\end{array}$ & $\begin{array}{l}128.28 \\
131.96 \\
152.71 \\
158.12 \\
283.05 \\
291.84\end{array}$ & $\begin{array}{l}\text { Feld, Smec, Waira, Phil, Cal } \\
\text { Feld, Smec, Phil, Waira, Anal, Cal } \\
\text { Waira, Feld, Phil, Anal, Smec } \\
\text { Waira, Feld, Phil, Anal, Cal } \\
\text { Feld, Smec, Cal } \\
\text { Feld, Smec, Cal }\end{array}$ \\
\hline $\begin{array}{l}\text { 126-788D- } \\
\text { 7R-CC, 7-9 }\end{array}$ & 280.14 & Feld, Smec, Phil, Cal \\
\hline $\begin{array}{l}\text { 126-792E- } \\
32 R-3,81-84 \\
37 R-4,57-61 \\
47 R-2,88-93 \\
50 R-3,114-118 \\
64 R-2,146-149 \\
67 R-3,98-102\end{array}$ & $\begin{array}{l}438.41 \\
487.27 \\
581.78 \\
611.74 \\
745.16 \\
775.18\end{array}$ & $\begin{array}{l}\text { Feld, Phil, Smec } \\
\text { Feld, Waira, Smec, Anal } \\
\text { Waira, Feld, Smec, Phil, Anal } \\
\text { Feld, Cristb, Smec } \\
\text { Waira, Smec, Anal, Feld } \\
\text { Feld, Smec, Cristb }\end{array}$ \\
\hline $\begin{array}{l}\text { 126-793B- } \\
\text { 20R-4, 91-95 } \\
26 \mathrm{R}-5,125-129 \\
41 \mathrm{R}-5,111-117 \\
49 \mathrm{R}-4,30-34 \\
56 \mathrm{R}-3,26-30 \\
59 \mathrm{R}-5,74-77 \\
64 \mathrm{R}-6,14-18\end{array}$ & $\begin{array}{r}774.91 \\
832.95 \\
977.73 \\
1052.10 \\
1118.26 \\
1150.34 \\
1199.54\end{array}$ & $\begin{array}{l}\text { Feld, Heulan, Smec } \\
\text { Waira, Feld, Smec, Phil, Anal } \\
\text { Heulan, Chabaz, Feld, Erion, Natrol } \\
\text { Heulan, Feld, Morden, Smec } \\
\text { Heulan, Feld, Smec, Morden } \\
\text { Waira, Herschel, Phil, Smec } \\
\text { Waira, Heulan, Morden, Smec, Feld }\end{array}$ \\
\hline
\end{tabular}

Notes: Feld $=$ feldspar, Smec $=$ smectite, Waira $=$ wairakite, Phil $=$ phillipsite, $\mathrm{Cal}=$ calcite, $\mathrm{Anal}=$ analcite $\mathrm{Cristb}=$ cristobalite, $\mathrm{Heulan}=$ heulandite, Chabaz $=$ chabazite, Erion $=$ erionite, Natrol $=$ natrolite, Morden $=$ morde nite, and Herschel $=$ herschelite.

\section{PETROGRAPHIC RESULTS}

\section{Backarc Sites 790 and 791}

Sites 790 and 791 are located in the central portion of the Sumisu Rift; Site 790 is situated on a structural high $2.4 \mathrm{~km}$ to the east of Site 791. Quaternary sections penetrated at these sites are predominantly composed of vitric silt, vitric sand, pumiceous sand, and burrowed nannofossil-rich clay and claystone (Taylor, Fujioka, et al., 1990). At Site 791, $834 \mathrm{~m}$ of Quaternary sediment overlie basement, whereas at Site 790,266 m of Quaternary sediment was drilled before reaching basement. The majority of sandy intervals at these sites are unconsolidated (see Marsaglia, this volume). The few lithified sandy units are bioturbated, poorly sorted, and matrix-rich (Table 2); these lithified units alternate with unlithified volcanic sands described by Marsaglia (this volume). Although the lithified matrix-rich samples exhibit no inter- or intraparticle cementation, they do show evidence of compaction and isolated secondary porosity. The secondary porosity results from the dissolution of feldspar microlites and glassy groundmass in volcanic lithic fragments (Table 2). Observed compaction features include crushed foraminifer tests and feldspar crystals. Severe diagenetic alteration of glass is present only in an unconsolidated sample taken from the volcanic "basement" (Sample 126-791B75R-1, 75-78 cm; see Marsaglia, this volume). This unconsolidated sample consists of disaggregated, diagenetically altered volcanic debris (Marsaglia, this volume). Many of the grains are zoned clay-mineral vesicle fillings (Marsaglia, this volume), similar to those observed in altered forearc basin sediments. This "sand" was probably artificially produced by drilling into and disaggregating a diagenetically altered volcaniclastic sand/tuff with high primary/secondary porosity. Similar textures were observed by the authors aboard the JOIDES Resolution, when altered lithified units (forearc sections) were physically disaggregated to make smear slides for shipboard description.

\section{Rift Flank Site 788}

Site 788 is located on the flank of the Sumisu Rift between two arc-related volcanos, Sumisu Jima and Torishima. The uppermost section (229 m) consists of Quaternary to Pliocene unconsolidated pumiceous gravels that gradually become lithified with depth. These gravels overlie a 145-m-thick section of indurated, Pliocene sedimentary rocks that vary in lithology from pumiceous conglomerate to claystone. The only diagenetic observation of note in sands from the unconsolidated units (see Marsaglia, this volume) is the presence of diagenetic opaques in Sample $126-788 \mathrm{C}-7 \mathrm{H}-1,30-33 \mathrm{~cm}$. These opaques occur as microspherules within vesicles of pumice fragments and as sand-size, loose, grapelike clusters of microspherules. They were identified as todorokite by shipboard scientists (Taylor, Fujioka, et al., 1990, pp. 97-126). This same sample also contains globular glass alteration products composed of clay minerals similar to the alteration products observed in more deeply buried forearc sections (see below).

The first consolidated sands occur within the transition zone from unconsolidated gravel to conglomerate at approximately $170 \mathrm{mbsf}$ (Table 2). Partially indurated pumiceous intervals containing drilling biscuits of lithified pumiceous coarse sand were sampled. These first indurated samples contain high inter-and intraparticle porosity. They are polymodal, consisting of coarse-sand-size, colorless pumice fragments and smaller dark lithic fragments. They appear to be lithified as a result of compaction and/or pressure welding (Plate 3, Figs. 1 and 2), accompanied by only minor cementation by phillipsite and minor clay-mineral rim cements (smectite and/or chlorite). The phillipsite cement takes the potassium stain and occurs as silt-size, spherical clusters of crystals attached to grains. Diagenetic reactions have produced incipient grain alteration at the grain boundaries and partial to complete dissolution of intermediate to basaltic brown glass fragments (Plate 3, Fig. 3; see Rodolfo et al. [this volume] for detailed chemical analyses of colorless, tan, and brown glass fragments). In contrast to the brown glass fragments, colorless rhyolitic glass fragments (see Rodolfo et al., this volume) show no evidence for dissolution, but occasionally are partially to wholly altered to green/ brown nonbirefringent clay minerals. This alteration is sometimes localized around mafic mineral grains or adjacent to fragments of black, mafic, tachylitic glass (Plate 3, Fig. 4). These clay-mineral alteration products are optically similar to the thin clay-mineral rim cements, which are also better developed in regions surrounding opaque grains (Plate 4, Fig. 1). There has also been some dissolution of calcareous foraminifers in these samples.

Lithified units below 240 mbsf show the effects of compaction (Plate 4, Fig. 2) and are cemented by variable amounts of zeolite (Plate 4, Fig. 2) and nonbirefringent to birefringent brown clay minerals. X-ray and SEM analysis of one sample indicates the presence of phillipsite and smectite (Table 1). Zeolite crystals petrographically observed in this sample take only the potassium stain or take both stains (implying that they are potassium- and calcium-bearing zeolites), but the potassium stain is more prominent. Some pumice grains have been altered to clay minerals (Plate 4, Fig. 3), and dissolution of brown glass is common (Plate 4, Fig. 4). Calcareous foraminifers (Plate 5, Fig. 1), and occasionally pyroxene and plagioclase grains, also show dissolution textures. Microcrystalline carbonate cement occurs in two samples (Table 2), primarily as minor overgrowths on foraminifer tests (Plate 4, Fig. 4).

\section{Forearc Sites (787, 792, and 793)}

\section{Quaternary-Miocene}

All Quaternary and Pliocene sands cored at the three Izu-Bonin forearc sites (787, 792, and 793) are unconsolidated (Marsaglia, this volume). A complete section of the Quaternary to Oligocene forearc basin fill was only cored at Site 792. Part of the Quaternary and the entire Pliocene to middle Miocene sedimentary sections were not cored at Site 793 because of time constraints. Coring at Site 793 was suspended at 100 mbsf until a depth of $586 \mathrm{mbsf}$, at which point an olivine diabase dike/sill was cored underlain by early to middle Miocene sediments. An extremely thin $(<50 \mathrm{~m})$ post-Oligocene section was present at Site 787 because of erosion/nondeposition within the Aoga Shima Canyon, where the site was located (Taylor, Fujioka, et al., 1990).

The first lithified forearc sandy sediments were recovered in bioturbated, muddy Miocene units at Sites 792 and 793 (Table 2). In 

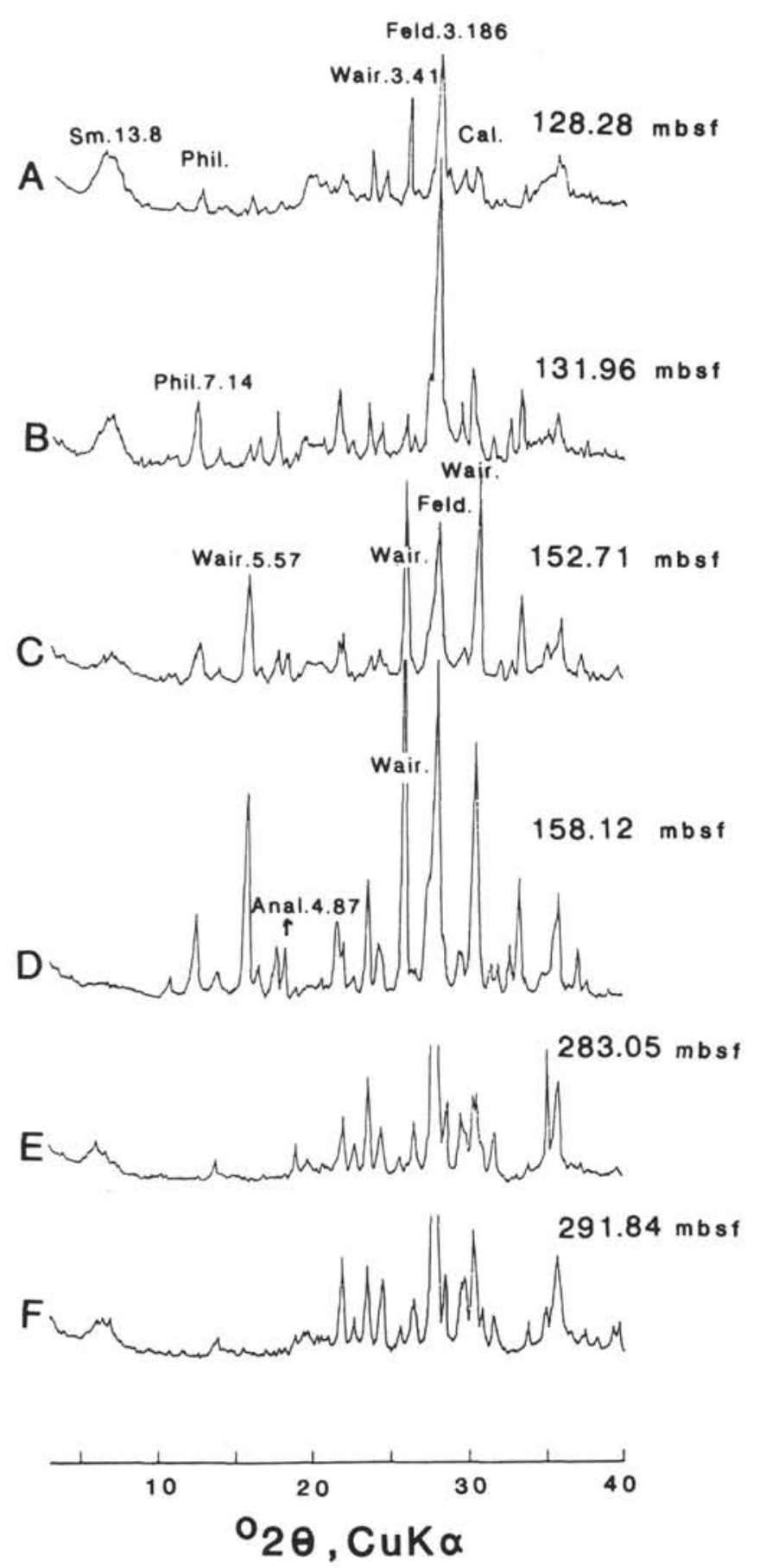

Figure 1. X-ray diffraction patterns for samples from Hole 787B. Sub-bottom depths of samples are indicated on right. A. Sample 126-787B-15R-1, 108-110 cm. B. Sample 126-787B-15R-4, 26-29 cm. C. Sample 126-787B-17R-5, 92-95 cm. D. Sample 126-787B-18R-2, 42-44 cm. E. Sample 126-787B-31R-1, $135-138 \mathrm{~cm}$. F. Sample 126-787B-32R-1, 54-56 cm. Feld = feldspar, $\mathrm{Sm}=$ smectite, Wair = wairakite, $\mathrm{Phil}=$ phillipsite, $\mathrm{Cal}=$ calcite , and $\mathrm{Anal}=$ analcite .

the Miocene section at Site 792, lithified matrix-rich sandy samples alternate with unconsolidated sands (sieved residues indicate that these samples are also matrix-rich). The matrices vary in composition from calcareous nannofossil-rich clay to silt-size volcanic detritus and clay. The lithified samples at both forearc sites show no evidence of cementation, only dissolution of brown glass (Table 2).

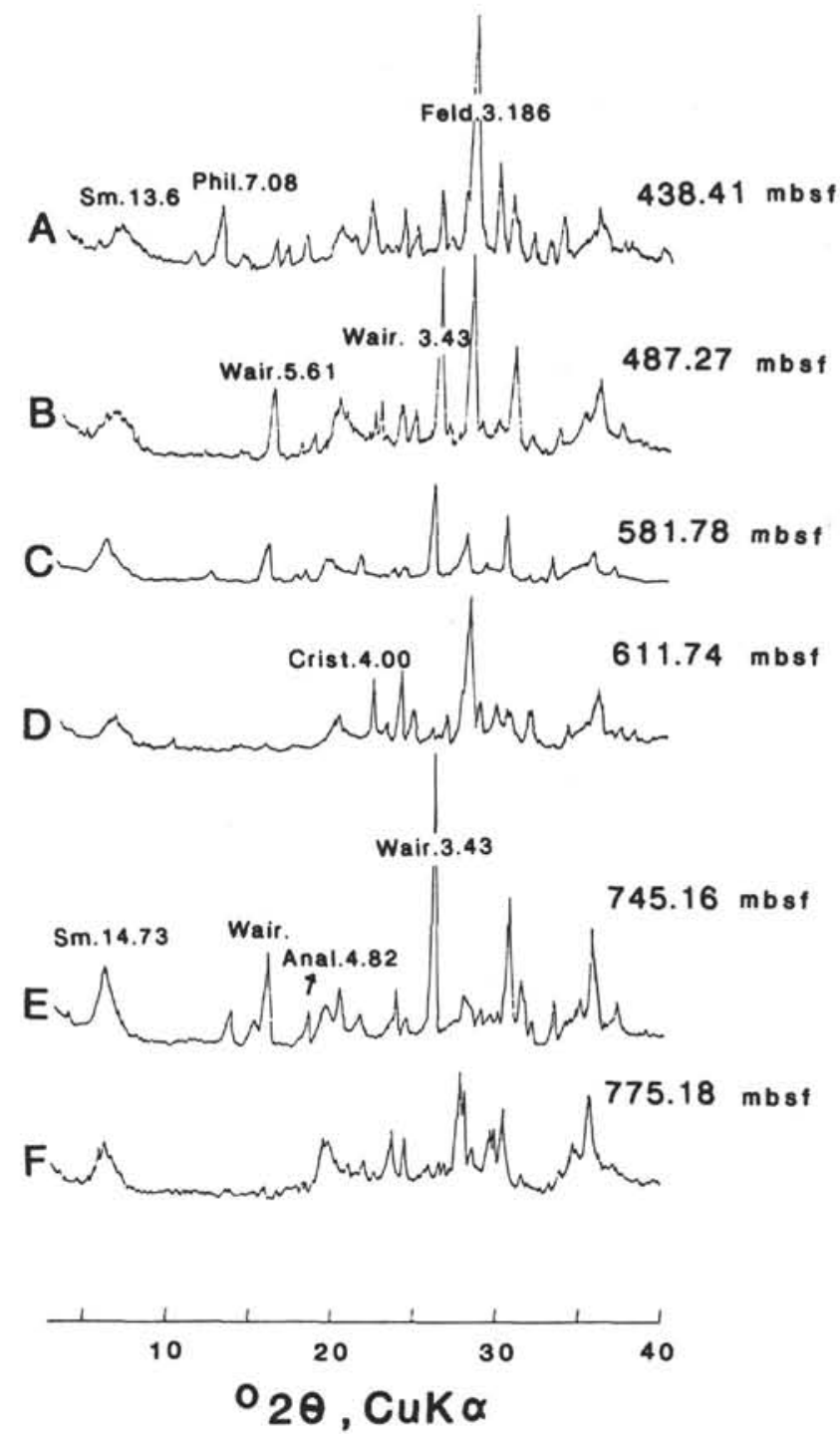

Figure 2. X-ray diffraction patterns for samples from Hole 792E. Sub-bottom depths of samples are indicated on right. A. Sample 126-792E-32R-3, 81-84 cm. B. Sample 126-792E-37R-4, 57-61 cm. C. Sample 126-792E-47R-2, 88-93 cm. D. Sample 126-792E-50R-3, 114-118 cm. E. Sample 126-792E-64R-2, 146-149 $\mathrm{cm}$. F. Sample 126-792E-67R-3, 98-102 cm. Abbreviations are as in Figure 1.

\section{Oligocene}

Intense diagenetic alteration of volcaniclastic forearc sands/sandstones is restricted to Oligocene volcaniclastic sediments at Sites 787, 792, and 793. Petrographic identification of the wide assortment of zeolite minerals present in these Oligocene samples (Table 1, Figs. 1-3, and Plates 1 and 2) was simplified for preliminary trend analysis by grouping these minerals (like feldspars) by stain type and intensity (zeolite categories in Table 2). Tentative correlations between type of stain and zeolite mineralogy are as follows: (1) unstained $(\mathrm{Na})=$ analcite, mordenite, or natrolite; $(2)$ red $(\mathrm{Ca})=$ heulandite, wairakite, or chabazite; (3) mixed $(\mathrm{Ca}, \mathrm{K}$ or $\mathrm{K}, \mathrm{Ca})=$ erionite, herschelite, or phillipsite; and (4) yellow $(\mathrm{K})=$ phillipsite/clinoptilolite. Mixing varies from heavy red/light yellow, to heavy yellow/light red, to light red and light yellow. In some instances, compositional zoning in pore-filling zeolites is manifested in the degree/intensity of stain. Pore-filling cement with similar optical properties was also seen to vary in composition from pore to pore or across a slide (not a result 
of local irregularities in staining process). Potassium-rich, yellowstained zeolites and mixed-stained, potassium- and calcium-bearing zeolites occur primarily as interparticle pore fillings, whereas zeolites with light and dark red stains and unstained zeolites occur as interand intraparticle cements (Plate 5, Figs. 2 and 3).

The clay-mineral cements in Oligocene forearc sandstones are primarily smectitic (various shades of brown and green), with lesser chlorite and other clay minerals (Table 1; also see table 3 [p. 341] and table 4 [p. 246] in Taylor, Fujioka, et al., 1990). These clays vary from nonbirefringent to first-order yellow birefringence (Plate 5, Figs. 2 and 3). In many thin sections, thick smectite rim cements are cracked because of dewatering of the smectitic clays during sample preparation. Other nonswelling, green clayrim cements (chlorite?) are unaffected by the drying process and maintain their fibroradiating rim morphology.

Carbonate cement rarely occurs as inter- and intraparticle porefilling crystals, especially in association with calcareous bioclastic debris. Other traces of carbonate cement occur as late-stage isolated crystals or crystal clusters in partially to wholly dissolved feldspar crystals (deeper sections of Site 792).

At Site 787, the shallowest Oligocene sample is matrix-rich and shows only minimal dissolution of brown glass and pyroxene crystals; however, it is directly underlain by extremely altered volcaniclastic sandstones. The degree of diagenetic alteration generally decreases down- section (Table 2), with an abrupt transition at approximately $170 \mathrm{mbsf}$ (Core 126-787B-19R). Samples from Cores 126-787B-14R through $-19 \mathrm{R}$ are cemented by both brown and green clay-mineral cements and a wide variety of zeolites, with up to four different zeolite compositions within the uppermost samples (Table 2). Below Core 126-787B-19R, pyroxene crystals rarely show evidence of dissolution, feldspar grains are fresh and show little to no evidence of dissolution, red-stained and unstained zeolites are not important constituents, and mixed (red/yellow) zeolites decrease in abundance. Clay-mineral cements and glass dissolution, and rare pyroxene dissolution occur in these lower cores. Coring at this site was suspended because of technical difficulties, and so the entire sedimentary section above basement was not penetrated.

A similar decrease in the intensity of diagenetic alteration/cementation can be seen at Site 792 (Table 2). There appears to be a rather abrupt decrease or change in diagenetic effects below Core 126-792E47R (approximately $590 \mathrm{mbsf}$ ). Unlike Site 787, diagenetic effects become more pronounced at the bottom of the hole (Cores 126-792E$62 \mathrm{R}$ to $-68 \mathrm{R}$; 729-782 mbsf) directly above basement. These lowermost samples are characterized by the presence of late-stage carbonate cements and possible authigenic potassium feldspar.

The diagenetic complexity of samples from Site 793 is apparent in Table 2. This site exhibits the widest variety of zeolite mineralogies (Table 1), and in many instances porosity has been totally destroyed

Table 2. Summary of petrographic observations, Sites 787, 788, 790, 791, 792, and 793.

\begin{tabular}{|c|c|c|c|c|c|c|c|c|c|c|c|c|c|c|c|c|c|}
\hline $\begin{array}{l}\text { Core, section, } \\
\text { interval }(\mathrm{cm})\end{array}$ & $\begin{array}{l}\text { Depth } \\
\text { (mbsf) }\end{array}$ & $\begin{array}{l}\text { Brn } \\
\text { cly }\end{array}$ & $\begin{array}{l}\text { Grn } \\
\text { cly }\end{array}$ & $\begin{array}{l}\text { Drk } \\
\text { red } \\
\text { zeo }\end{array}$ & $\begin{array}{l}\text { Pal } \\
\text { red } \\
\text { zeo }\end{array}$ & $\begin{array}{l}\text { Red } \\
\text { yel } \\
\text { zeo }\end{array}$ & $\begin{array}{l}\text { Yel } \\
\text { zeo }\end{array}$ & $\begin{array}{l}\text { Uns } \\
\text { zeo }\end{array}$ & $\begin{array}{l}\text { Car } \\
\text { cem }\end{array}$ & $\begin{array}{l}\text { Dis } \\
\text { brn } \\
\text { gls }\end{array}$ & $\begin{array}{l}\text { Dis } \\
\text { pyr }\end{array}$ & $\begin{array}{l}\text { Fel } \\
\text { dis }\end{array}$ & $\begin{array}{l}\text { Fel } \\
\text { alt } \\
\text { zeo }\end{array}$ & $\begin{array}{l}\text { Cly } \\
\text { fil } \\
\text { gls }\end{array}$ & $\begin{array}{l}\text { Dis } \\
\text { yel } \\
\text { zeo }\end{array}$ & Por & $\begin{array}{l}\text { Qtz } \\
\text { cem }\end{array}$ \\
\hline $\begin{array}{l}\text { 126-787B- } \\
\text { 9R-2, 47-50 } \\
14 \mathrm{R}-2,69-73 \\
14 \mathrm{R}-3,72-76 \\
15 \mathrm{R}-1,108-111 \\
15 \mathrm{R}-3,52-54 \\
15 \mathrm{R}-4,26-29 \\
16 \mathrm{R}-1,46-49 \\
16 \mathrm{R}-5,35-38 \\
17 \mathrm{R}-4,10-12 \\
17 \mathrm{R}-5,21-22 \\
17 \mathrm{R}-5,92-95 \\
18 \mathrm{R}-1,17-20 \\
18 \mathrm{R}-2,42-44 \\
18 \mathrm{R}-3,141-145 \\
19 \mathrm{R}-3,31-34 \\
19 \mathrm{R}-6,45-48 \\
20 \mathrm{R}-1,15-16 \\
20 \mathrm{R}-4,20-23 \\
21 \mathrm{R}-1,65-69 \\
22 \mathrm{R}-1,47-50 \\
23 \mathrm{R}-4,18-21 \\
24 \mathrm{R}-6,91-94 \\
25 \mathrm{R}-1,119-122 \\
25 \mathrm{R}-4,10-13 \\
26 \mathrm{R}-2,88-91 \\
26 \mathrm{R}-5,19-22 \\
27 \mathrm{R}-1,77-80 \\
27 \mathrm{R}-\mathrm{CC}, 0-3 \\
29 \mathrm{R}-2,77-80 \\
30 \mathrm{R}-3,62-65 \\
31 \mathrm{R}-1,135-138 \\
32 \mathrm{R}-1,54-56 \\
33 \mathrm{R}-2,68-71 \\
34 \mathrm{R}-1,32-34\end{array}$ & $\begin{array}{l}70.97 \\
119.69 \\
121.22 \\
128.28 \\
130.72 \\
131.96 \\
137.26 \\
143.15 \\
151.10 \\
152.71 \\
153.42 \\
156.37 \\
158.12 \\
160.61 \\
169.11 \\
173.75 \\
175.65 \\
178.70 \\
185.75 \\
197.27 \\
209.78 \\
222.51 \\
224.89 \\
228.30 \\
235.78 \\
239.59 \\
243.77 \\
248.75 \\
264.67 \\
275.62 \\
283.05 \\
291.84 \\
302.98 \\
310.82\end{array}$ & $\begin{array}{l}\mathrm{X} \\
\mathrm{X} \\
\mathrm{X}\end{array}$ & $\begin{array}{l}X \\
X \\
X \\
\\
X \\
X \\
X \\
X \\
X \\
X \\
X \\
x \\
X \\
X \\
X \\
X \\
X \\
X \\
X \\
x \\
X \\
X \\
X \\
X \\
X\end{array}$ & $\begin{array}{l}\mathrm{X} \\
\mathrm{X} \\
\mathrm{X} \\
\mathrm{X}\end{array}$ & $\begin{array}{l}\text { X } \\
\text { X } \\
\text { X } \\
\text { X } \\
\text { X } \\
\text { Tr } \\
\text { X } \\
\text { X } \\
\text { X } \\
\text { X } \\
\text { X } \\
\text { X } \\
\text { X }\end{array}$ & $\begin{array}{l}\mathrm{X} \\
\mathrm{X} \\
\mathrm{X} \\
\mathrm{X} \\
\mathrm{X} \\
\mathrm{X} \\
\mathrm{X} \\
\mathrm{X} \\
\mathrm{X} \\
\mathrm{X} \\
\mathrm{X} \\
\mathrm{X} \\
\mathrm{X} \\
\mathrm{X} \\
\mathrm{X} \\
\\
\mathrm{X} \\
\mathrm{X} \\
\mathrm{X} \\
\mathrm{X} \\
\mathrm{X} \\
\mathrm{X} \\
? \\
\mathrm{X} \\
\mathrm{X}\end{array}$ & $\begin{array}{l}\underset{X}{X} \\
\text { X } \\
\\
X \\
\\
X \\
\text { X } \\
\text { X } \\
\text { X } \\
\text { X }\end{array}$ & $\begin{array}{l}\mathrm{X} \\
\mathrm{X}\end{array}$ & $\mathrm{X}$ & $\begin{array}{l}\mathrm{X} \\
\mathrm{X} \\
\mathrm{X} \\
\mathrm{X} \\
\mathrm{X} \\
\mathrm{X} \\
\mathrm{X} \\
\mathrm{X} \\
\mathrm{X} \\
\mathrm{X} \\
\mathrm{X} \\
\mathrm{X} \\
\mathrm{X} \\
\mathrm{X} \\
\mathrm{X} \\
\mathrm{X} \\
\mathrm{X} \\
\mathrm{X} \\
\mathrm{X} \\
\mathrm{X} \\
\mathrm{X} \\
\mathrm{X} \\
\mathrm{X} \\
\mathrm{X} \\
\mathrm{X} \\
\mathrm{X} \\
\mathrm{X} \\
\mathrm{X} \\
\mathrm{X} \\
\mathrm{X} \\
\mathrm{X} \\
\mathrm{X}\end{array}$ & $\begin{array}{l}\mathrm{X} \\
\mathrm{X} \\
\mathrm{X} \\
\mathrm{X} \\
\mathrm{X} \\
\mathrm{X} \\
\mathrm{X} \\
\mathrm{X} \\
\mathrm{X} \\
\mathrm{X}\end{array}$ & $\begin{array}{l}\text { X } \\
\text { X } \\
\text { X } \\
\text { X } \\
\text { X } \\
\text { X } \\
\\
\text { X } \\
\text { Tr } \\
\underset{X}{X} \\
\underset{X}{X} \\
\\
T r \\
T r\end{array}$ & $\begin{array}{l}\mathrm{X} \\
\mathrm{X} \\
\mathrm{X} \\
\mathrm{X} \\
\mathrm{X} \\
\mathrm{X} \\
\mathrm{X} \\
\mathrm{X} \\
\mathrm{X} \\
\mathrm{X} \\
\mathrm{X}\end{array}$ & $\begin{array}{l}\mathrm{X} \\
\mathrm{X} \\
\mathrm{X} \\
\mathrm{X} \\
\mathrm{X} \\
\mathrm{X} \\
\mathrm{X} \\
\mathrm{X} \\
\mathrm{X} \\
\mathrm{X} \\
\mathrm{X} \\
\mathrm{X} \\
\mathrm{X} \\
\mathrm{X} \\
\mathrm{X} \\
\mathrm{X} \\
\mathrm{X} \\
\mathrm{X} \\
\mathrm{X} \\
\mathrm{X} \\
\mathrm{X} \\
\mathrm{X}\end{array}$ & & $\begin{array}{l}\mathrm{X} \\
\mathrm{X} \\
\mathrm{X} \\
\mathrm{X} \\
\mathrm{X} \\
\mathrm{X} \\
\mathrm{Tr} \\
\mathrm{X} \\
\mathrm{X} \\
\mathrm{X} \\
\mathrm{X} \\
\mathrm{X} \\
\mathrm{X} \\
\mathrm{X} \\
\mathrm{X} \\
\mathrm{X} \\
\mathrm{X} \\
\mathrm{X} \\
\mathrm{X} \\
\mathrm{X} \\
\mathrm{X} \\
\mathrm{X} \\
\mathrm{X} \\
\mathrm{X} \\
\mathrm{X} \\
\mathrm{X} \\
\mathrm{X} \\
\mathrm{X} \\
\mathrm{X} \\
\mathrm{X} \\
\mathrm{X} \\
\mathrm{X}\end{array}$ & \\
\hline $\begin{array}{l}126-788 \mathrm{C}- \\
19 \mathrm{H}-1,69-71 \\
20 \mathrm{H}-2,40-42\end{array}$ & $\begin{array}{l}172.89 \\
183.60\end{array}$ & $\begin{array}{l}\mathrm{Tr} \\
\mathrm{Tr}\end{array}$ & & & & & $\mathrm{Tr}$ & & & $\underset{\mathrm{X}}{\mathrm{X}}$ & & & & & & $\begin{array}{l}\mathrm{X} \\
\mathrm{X}\end{array}$ & \\
\hline $\begin{array}{l}\text { 126-788D- } \\
\text { 4R-1, 27-33 } \\
\text { 5R-1, 42-45 } \\
\text { 6R-1, 24-26 } \\
\text { 7R-CC, 7-9 } \\
\text { 8R-1, 29-32 } \\
\text { 9R-1, 101-105 } \\
11 \mathrm{R}-1,17-20 \\
\text { 12R-1, 17-19 }\end{array}$ & $\begin{array}{l}248.77 \\
258.62 \\
268.14 \\
280.14 \\
287.49 \\
297.91 \\
316.17 \\
325.77\end{array}$ & $\begin{array}{l}\mathrm{Tr} \\
\mathrm{Tr} \\
\mathrm{Tr} \\
\mathrm{Tr} \\
\mathrm{Tr} \\
\mathrm{Tr} \\
\mathrm{Tr} \\
\mathrm{Tr}\end{array}$ & & & $\mathrm{X}$ & $\begin{array}{l}\mathrm{X} \\
\mathrm{X} \\
\mathrm{X} \\
\mathrm{Tr} \\
\mathrm{X} \\
? \\
?\end{array}$ & $\begin{array}{l}\mathrm{X} \\
\mathrm{Tr} \\
\mathrm{X} \\
\mathrm{X} \\
\\
\mathrm{X} \\
?\end{array}$ & & $\mathrm{Tr}$ & $\begin{array}{l}\mathrm{X} \\
\mathrm{X} \\
\mathrm{X} \\
\mathrm{X} \\
\mathrm{X} \\
\mathrm{X} \\
\mathrm{X} \\
\mathrm{X}\end{array}$ & $\mathrm{X}$ & $\mathrm{X}$ & & & & $\begin{array}{l}\mathrm{X} \\
\mathrm{X} \\
\mathrm{X} \\
\mathrm{X} \\
\mathrm{X} \\
\mathrm{X} \\
\underset{\mathrm{X}}{\mathrm{X}}\end{array}$ & \\
\hline $\begin{array}{l}\text { 126-791A- } \\
46 \mathrm{X}-\mathrm{CC}, 8-18\end{array}$ & 418.88 & * & & & & & & & & $\mathrm{X}$ & & & & & & & \\
\hline $\begin{array}{l}\text { 126-791B } \\
\text { 44R-1, 22-24 } \\
\text { 44R-1, 101-104 } \\
\text { 47R-1, 36-39 }\end{array}$ & $\begin{array}{l}800.92 \\
801.71 \\
829.96\end{array}$ & * & & & & & & & & $\begin{array}{l}\mathrm{X} \\
\mathrm{X} \\
\mathrm{X}\end{array}$ & & $\underset{\mathrm{X}}{\mathrm{X}}$ & & & & & \\
\hline
\end{tabular}


by pore-filling cements (Table 2). Although there are downhole changes in the assemblages of diagenetic minerals and grain alteration/dissolution, no distinct changes in the intensity of diagenesis within these samples is apparent.

\section{GRAIN DISSOLUTION AND ALTERATION}

Vitric components present in Leg 126 sandstones have reacted differently during diagenesis as a function of their chemical composition. The black, opaque-rich groundmass of mafic tachylitic fragments appears to be grossly unaffected by diagenetic processes, even within deeply buried Oligocene forearc sections. In contrast, $\tan$ to brown, intermediate to mafic, glassy fragments (see Rodolfo et al., this volume) readily undergo dissolution. Colorless, rhyolitic (see Rodolfo, this volume) glassy fragments show no evidence for dissolution but are locally altered to clay minerals at Sites 787, 788, 792, and 793.

Hydrothermal experiments on rhyolitic glass indicate that at high temperatures $\left(200^{\circ} \mathrm{C}\right)$, rhyolitic glass does not recrystallize but acts as $\mathrm{Na}-\mathrm{K}$ ion exchanger (Shiraki and Iiyama, 1990). Perhaps this characteristic of rhyolitic glass plays a role in its direct alteration (solid-solid transformation) to clay minerals in some Leg 126 samples. The cation flux is dependent on the cooling rate of the glass: quenched glass fixes potassium, whereas slowly cooled glass fixes sodium (Shiraki and Iiyama, 1990). Therefore, variations in cooling history might explain why some rhyolitic vitric fragments alter more readily to clay minerals (i.e., Plate 4 , Fig. 3 ).

Tan/brown intermediate to mafic glass is chemically less stable than rhyolitic glass and undergoes dissolution more readily, resulting in secondary porosity. Although tan/brown glass is readily dissolved during early diagenesis, the pyroxene and feldspar microlites within these grains appear to be minimally affected. Pyroxene and plagioclase dissolution are only commonplace in Oligocene forearc sections. Our observations agree with the findings of Boles and Coombs (1977) that glass alters before plagioclase in volcaniclastic sediments.

Porosity/cement relationships in Leg 126 volcaniclastic sandstones suggest that the "zeolitization" of glass and feldspar is a dissolution-precipitation reaction. Porosity is first created by the early dissolution of intermediate to mafic brown glassy fragments (see Rodolfo, this volume), and then destroyed by the later precipitation of smectite and/or zeolite cements. The smectite precipitation must occur after the dissolution of glass but before the dissolution of feldspar. Clay cements are observed within dissolved vitric grains; however, clay cement does not infill secondary porosity in feldspar grains. In all cases, clay-mineral cements precede zeolite cementation (all compositions).

Our findings differ from generalizations made by Fisher and Schmincke (1984), who suggest that, during early diagenesis, basaltic glass alters to smectite whereas rhyolitic glass simply undergoes minor chemical changes. According to Fisher and Schmincke (1984), first basaltic glass is altered to smectite, and then rhyolitic glass is dissolved and secondary minerals are precipitated in the resultant pore spaces. We observe the opposite trend in Leg 126 volcaniclastic sandstones, where intermediate to mafic, brown glass is dissolved, and colorless, rhyolitic glass is altered to clay minerals.

\section{DISCUSSION}

Despite the thick accumulation of Quaternary volcaniclastic sediment in the Sumisu Rift basin, diagenesis within the basin fill is

Table 2 (continued).

\begin{tabular}{|c|c|c|c|c|c|c|c|c|c|c|c|c|c|c|c|c|c|}
\hline $\begin{array}{l}\text { Core, section, } \\
\text { interval }(\mathrm{cm})\end{array}$ & $\begin{array}{l}\text { Depth } \\
\text { (mbsf) }\end{array}$ & $\begin{array}{l}\text { Brn } \\
\text { cly }\end{array}$ & $\begin{array}{l}\text { Grn } \\
\text { cly }\end{array}$ & $\begin{array}{l}\text { Drk } \\
\text { red } \\
\text { zeo }\end{array}$ & $\begin{array}{l}\text { Pal } \\
\text { red } \\
\text { zeo }\end{array}$ & $\begin{array}{l}\text { Red } \\
\text { yel } \\
\text { zeo }\end{array}$ & $\begin{array}{l}\text { Yel } \\
\text { zeo }\end{array}$ & $\begin{array}{l}\text { Uns } \\
\text { zeo }\end{array}$ & $\begin{array}{l}\text { Car } \\
\text { cem }\end{array}$ & $\begin{array}{c}\text { Dis } \\
\text { brn } \\
\text { gls }\end{array}$ & $\begin{array}{l}\text { Dis } \\
\text { pyr }\end{array}$ & $\begin{array}{l}\text { Fel } \\
\text { dis }\end{array}$ & $\begin{array}{c}\text { Fel } \\
\text { alt } \\
\text { zeo }\end{array}$ & $\begin{array}{c}\text { Cly } \\
\text { fil } \\
\text { gls }\end{array}$ & $\begin{array}{l}\text { Dis } \\
\text { yel } \\
\text { zeo }\end{array}$ & Por & $\begin{array}{l}\text { Qtz } \\
\text { cem }\end{array}$ \\
\hline 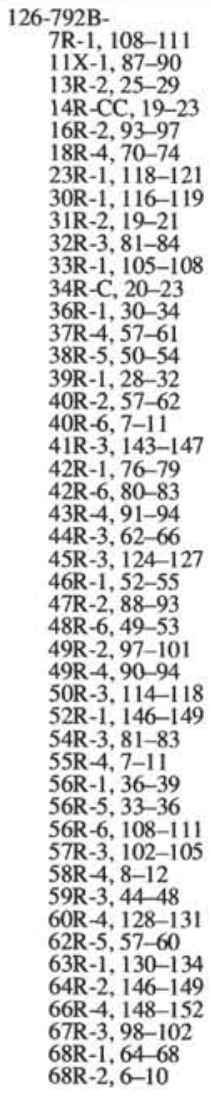 & $\begin{array}{l}194.48 \\
232.77 \\
252.95 \\
26.90 \\
282.63 \\
305.10 \\
348.78 \\
416.36 \\
428.59 \\
438.41 \\
445.25 \\
458.60 \\
473.50 \\
487.27 \\
499.00 \\
502.48 \\
513.97 \\
519.47 \\
526.03 \\
531.96 \\
539.50 \\
546.01 \\
553.92 \\
564.24 \\
570.22 \\
581.78 \\
596.59 \\
600.27 \\
603.20 \\
611.74 \\
628.26 \\
649.91 \\
660.27 \\
665.76 \\
671.73 \\
673.98 \\
679.12 \\
689.38 \\
699.84 \\
709.78 \\
729.47 \\
733.80 \\
745.16 \\
767.48 \\
775.18 \\
781.54 \\
782.46\end{array}$ & $\begin{array}{l}\mathrm{X} \\
\mathrm{X} \\
\mathrm{X} \\
\mathrm{X} \\
\mathrm{X} \\
\mathrm{X}\end{array}$ & $\begin{array}{l}X \\
X \\
X \\
X \\
X \\
X \\
X \\
X \\
X \\
T Y \\
X \\
X \\
X \\
X \\
X \\
X \\
X \\
X \\
X \\
X \\
X \\
X \\
X\end{array}$ & $\begin{array}{l}\mathrm{Tr} \\
\mathrm{X} \\
\mathrm{X} \\
\mathrm{X} \\
\mathrm{X} \\
\mathrm{X} \\
\mathrm{X}\end{array}$ & $\begin{array}{l}\mathrm{X} \\
\mathrm{X} \\
\mathrm{X} \\
\mathrm{X} \\
\mathrm{X} \\
\mathrm{X} \\
\mathrm{X} \\
\mathrm{X} \\
\mathrm{X} \\
\mathrm{X} \\
\mathrm{X} \\
\mathrm{X} \\
\mathrm{X} \\
\mathrm{X} \\
\mathrm{X} \\
\mathrm{X}\end{array}$ & $\begin{array}{l}X \\
X \\
X \\
X \\
X \\
X \\
X \\
X \\
X \\
X \\
X \\
X \\
X \\
X \\
X \\
X \\
X \\
X \\
X \\
X \\
X \\
X\end{array}$ & $\begin{array}{l}\underset{X}{X} \\
\underset{X}{X} \\
X \\
X \\
X \\
\text { X } \\
\text { X } \\
\text { X }\end{array}$ & $\begin{array}{l}X \\
X \\
X \\
X \\
X \\
X\end{array}$ & $\begin{array}{l}\text { X } \\
\text { X } \\
\\
\text { Tr } \\
\text { Tr } \\
\\
\underset{X}{X}\end{array}$ & 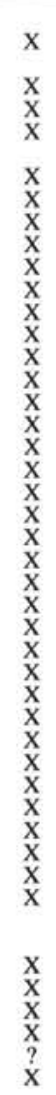 & $\begin{array}{l}X \\
X \\
X \\
X \\
X \\
X \\
X \\
X \\
X \\
\\
X \\
X \\
X \\
X \\
X \\
X \\
X \\
X \\
X \\
X \\
X \\
X \\
X \\
X \\
X \\
X \\
X \\
X \\
X \\
X \\
X \\
X \\
X \\
X \\
X \\
X \\
X \\
X\end{array}$ & $\begin{array}{l}\mathrm{X} \\
\mathrm{X} \\
\mathrm{X} \\
\mathrm{X} \\
\mathrm{X} \\
\mathrm{X} \\
\mathrm{X} \\
\mathrm{X} \\
\mathrm{X} \\
\mathrm{X} \\
\mathrm{X} \\
\mathrm{X} \\
\mathrm{X} \\
\mathrm{X} \\
\mathrm{X} \\
\mathrm{X} \\
\mathrm{X} \\
\mathrm{X} \\
\mathrm{X} \\
\mathrm{X} \\
\mathrm{X} \\
\mathrm{X} \\
\mathrm{X}\end{array}$ & $\begin{array}{l}\mathrm{X} \\
\mathrm{X} \\
\mathrm{X} \\
\mathrm{X} \\
\mathrm{X} \\
\mathrm{X} \\
\mathrm{X} \\
\mathrm{X} \\
\mathrm{X} \\
\mathrm{X} \\
\mathrm{X} \\
\mathrm{X} \\
\mathrm{X} \\
\mathrm{X} \\
\mathrm{X} \\
\mathrm{Tr} \\
\mathrm{Tr} \\
\mathrm{Tr} \\
\mathrm{X} \\
\mathrm{X}\end{array}$ & $\begin{array}{c}X \\
X \\
X \\
X \\
X \\
X \\
X \\
X \\
X \\
X \\
X \\
X \\
X \\
X \\
X \\
X \\
X \\
X \\
X \\
X \\
X \\
X \\
X \\
X \\
X \\
X \\
X \\
X \\
X \\
X \\
X \\
X \\
X \\
X\end{array}$ & $\begin{array}{l}\mathrm{X} \\
\mathrm{X} \\
\mathrm{X}\end{array}$ & $\begin{array}{l}\text { X } \\
X \\
X \\
X \\
X \\
X \\
X \\
X \\
X \\
X \\
\mathrm{Tr} \\
X \\
X \\
X \\
? \\
? \\
X \\
X \\
X \\
X \\
X \\
X \\
X \\
X \\
X \\
X \\
X \\
X \\
X \\
X \\
X \\
X \\
X \\
X \\
X \\
T \\
T \\
T r \\
X \\
T \\
X \\
X \\
X \\
X\end{array}$ & $\mathrm{X}$ \\
\hline
\end{tabular}


Table 2 (continued).

\begin{tabular}{|c|c|c|c|c|c|c|c|c|c|c|c|c|c|c|c|c|c|}
\hline $\begin{array}{l}\text { Core, section, } \\
\text { interval }(\mathrm{cm})\end{array}$ & $\begin{array}{l}\text { Depth } \\
\text { (mbsf) }\end{array}$ & $\begin{array}{l}\text { Brn } \\
\text { cly }\end{array}$ & $\begin{array}{l}\text { Grn } \\
\text { cly }\end{array}$ & $\begin{array}{l}\text { Drk } \\
\text { red } \\
\text { zeo }\end{array}$ & $\begin{array}{l}\text { Pal } \\
\text { red } \\
\text { zeo }\end{array}$ & $\begin{array}{l}\text { Red } \\
\text { yel } \\
\text { zeo }\end{array}$ & $\begin{array}{l}\text { Yel } \\
\text { zeo }\end{array}$ & $\begin{array}{l}\text { Uns } \\
\text { zeo }\end{array}$ & $\begin{array}{l}\mathrm{Car} \\
\mathrm{cem}\end{array}$ & $\begin{array}{c}\text { Dis } \\
\text { brn } \\
\text { gls }\end{array}$ & $\begin{array}{l}\text { Dis } \\
\text { pyr }\end{array}$ & $\begin{array}{l}\text { Fel } \\
\text { dis }\end{array}$ & $\begin{array}{l}\text { Fel } \\
\text { alt } \\
\text { zeo }\end{array}$ & $\begin{array}{l}\text { Cly } \\
\text { fil } \\
\text { gls }\end{array}$ & $\begin{array}{l}\text { Dis } \\
\text { yel } \\
\text { zeo }\end{array}$ & Por & $\begin{array}{l}\text { Qtz } \\
\text { cem }\end{array}$ \\
\hline \multicolumn{18}{|l|}{ 126-793B- } \\
\hline $3 R-1,66-70$ & 604.96 & * & & & & & & & & $\mathrm{x}$ & & & & & & & \\
\hline $9 \mathrm{R}-1,3-4$ & 662.23 & * & & & & & & & & & & & & & & & \\
\hline $10 \mathrm{R}-1,108-111$ & 672.88 & * & & & & & & & & & & & & & & & \\
\hline 10R-2, 13-16 & 673.43 & * & & & & & & & & $\mathrm{x}$ & & & & & & & \\
\hline IIR-1, 104-107 & 682.44 & $*$ & & & & & & & & $\hat{x}$ & & & & & & & \\
\hline $14 \mathrm{R}-1,125-127$ & 711.65 & $*$ & & & & & & & & & & & & & & & \\
\hline $15 \mathrm{R}-1,85-88$ & 720.95 & $*$ & & & & & & & & & & & & & & & \\
\hline $16 \mathrm{R}-2,83-86$ & 732.03 & * & & & & & & & & $\mathrm{x}$ & & & & & & & \\
\hline $19 \mathrm{R}-4,47-51$ & 763.37 & & $\mathrm{x}$ & $\mathrm{x}$ & $\mathrm{x}$ & $\mathrm{x}$ & $\mathrm{x}$ & & $\mathrm{x}$ & $\hat{x}$ & & & $\mathrm{x}$ & $\mathrm{x}$ & $\mathrm{X}$ & $\mathrm{x}$ & \\
\hline $20 \mathrm{R}-4,91-95$ & 774.91 & & $\hat{\mathrm{x}}$ & $\hat{\mathrm{x}}$ & $\mathrm{A}$ & 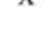 & 凡 & $\mathrm{x}$ & 人 & $\hat{x}$ & $\hat{\mathrm{x}}$ & $\hat{\mathrm{x}}$ & $\hat{x}$ & $\hat{\mathrm{x}}$ & A & $\hat{\mathrm{x}}$ & \\
\hline $2 \mathrm{IR}-2,109-112$ & $\begin{array}{l}780.29 \\
780.95\end{array}$ & & $\hat{T}$ & $\hat{\mathrm{x}}$ & & & & 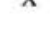 & & $\hat{x}$ & $\hat{\mathrm{x}}$ & $\hat{\mathrm{x}}$ & $\hat{\mathrm{X}}$ & $\mathrm{x}$ & & $\mathrm{Tr}_{\mathrm{X}}^{\mathrm{T}}$ & \\
\hline $\begin{array}{l}21 \mathrm{R}-3,25-28 \\
22 \mathrm{R}-3,33-36\end{array}$ & $\begin{array}{l}780.95 \\
790.63\end{array}$ & $x$ & $\mathrm{Tr}$ & $\stackrel{X}{x}$ & $\mathrm{x}$ & $\mathrm{x}$ & & $x$ & & $\begin{array}{l}X \\
x \\
x\end{array}$ & $\stackrel{X}{x}$ & $\begin{array}{l}x \\
x\end{array}$ & $\mathrm{x}$ & $\begin{array}{l}X \\
x \\
-1\end{array}$ & & $\stackrel{\hat{X}}{\mathrm{X}}$ & \\
\hline $23 R-4,9-12$ & 801.59 & $\hat{x}$ & $\hat{x}$ & $\hat{\mathrm{x}}$ & $\hat{\mathrm{x}}$ & & & $\hat{x}$ & & $\hat{x}$ & $\hat{\mathrm{x}}$ & $\hat{\mathrm{x}}$ & $\hat{\mathrm{X}}$ & & & $\hat{\operatorname{Tr}}$ & \\
\hline $24 \mathrm{R}-2,5-9$ & 808.25 & $\widehat{\operatorname{Tr}}$ & $\hat{x}$ & $\hat{x}$ & & $\mathrm{x}$ & & $\lambda$ & & $\hat{\mathrm{X}}$ & & $\hat{\mathrm{x}}$ & $\hat{\mathrm{x}}$ & & & $?$ & \\
\hline $25 \mathrm{R}-1,147-151$ & 817.87 & & $\hat{x}$ & $\hat{x}$ & & $\hat{x}$ & & $\mathrm{x}$ & & $\hat{x}$ & & $\hat{\mathrm{x}}$ & $\hat{x}$ & $\mathrm{x}$ & & $\mathrm{Tr}$ & \\
\hline $26 \mathrm{R}-5,125-129$ & 832.95 & $\mathrm{x}$ & $\hat{x}$ & $\hat{x}$ & $\mathrm{x}$ & $\hat{x}$ & & $\hat{x}$ & & $\hat{x}$ & & $\hat{\mathrm{x}}$ & $\hat{x}$ & $\hat{x}$ & & $\operatorname{Tr}$ & \\
\hline $27 \mathrm{R}-6,35-40$ & 843.25 & $\hat{\mathrm{x}}$ & $\hat{x}$ & $\hat{x}$ & $\hat{x}$ & $\mathrm{~A}$ & & & & $\hat{x}$ & & $\hat{\mathrm{x}}$ & $\hat{\mathrm{x}}$ & $\widehat{x}$ & & $\mathrm{X}$ & \\
\hline $28 \mathrm{R}-6,33-37$ & 852.93 & $\hat{\mathrm{x}}$ & $\hat{x}$ & $\mathrm{X}$ & $\mathrm{x}$ & $\mathrm{x}$ & & $\mathrm{x}$ & & $\hat{x}$ & $\mathrm{x}$ & $\mathrm{x}$ & $\hat{\mathrm{x}}$ & $\mathrm{x}$ & $\mathrm{x}$ & $\hat{\mathrm{x}}$ & \\
\hline $29 R-5,109-112$ & 861.79 & $\ddot{x}$ & $\mathrm{x}$ & $\mathrm{x}$ & $\mathrm{x}$ & & & & & $x$ & $?$ & $\mathrm{x}$ & $\mathrm{x}$ & $\mathrm{x}$ & & $\hat{\mathrm{x}}$ & \\
\hline $30 \mathrm{R}-3,25-29$ & 867.65 & $\mathrm{X}$ & $\mathrm{X}$ & & $\mathrm{X}$ & $\mathrm{X}$ & & & & $\mathrm{X}$ & & $\mathrm{X}$ & $\mathrm{X}$ & $\mathrm{x}$ & & $\operatorname{Tr}$ & \\
\hline $31 R-5,113-117$ & 881.13 & & $\mathrm{Tr}$ & $\mathrm{x}$ & $\mathrm{x}$ & $\mathrm{x}$ & & $\mathrm{X}$ & & $\mathrm{x}$ & & $\mathrm{x}$ & $\mathrm{X}$ & $\mathrm{X}$ & & $\mathrm{Tr}$ & \\
\hline $32 \mathrm{R}-1,39-43$ & 884.09 & & $\mathrm{x}$ & $\mathrm{x}$ & $\mathrm{x}$ & $\mathrm{x}$ & & & & $\mathrm{X}$ & & $\mathrm{x}$ & $\mathrm{x}$ & $\mathrm{Tr}$ & & $\mathrm{Tr}$ & \\
\hline $33 R-7,82-85$ & 902.82 & & $\mathrm{Tr}$ & $\mathrm{X}$ & & $\mathrm{x}$ & & & & $x$ & & $\mathrm{x}$ & $\mathrm{x}$ & $\mathrm{Tr}$ & & $\mathrm{Tr}$ & \\
\hline $34 \mathrm{R}-2,44-47$ & 904.94 & & $\ddot{x}$ & $\mathrm{x}$ & $\mathrm{x}$ & $\hat{x}$ & & & & $\hat{\mathrm{x}}$ & & $\hat{x}$ & $\hat{x}$ & $\mathrm{Tr}$ & & $\mathrm{Tr}$ & \\
\hline $35 \mathrm{R}-2,139-142$ & 915.49 & $\mathrm{X}$ & $\mathrm{x}$ & $\mathrm{x}$ & $\mathrm{x}$ & $\mathrm{x}$ & & & & $\hat{\mathrm{x}}$ & & $\hat{\mathrm{x}}$ & $\hat{\mathrm{x}}$ & $\mathrm{x}$ & & $\mathrm{Tr}$ & \\
\hline $36 \mathrm{R}-6,103-107$ & 930.83 & $\tilde{x}$ & $\mathrm{x}$ & $\mathrm{x}$ & $\mathrm{x}$ & $x$ & & & & $\hat{\mathrm{x}}$ & & $\hat{x}$ & $\hat{\mathrm{x}}$ & $\hat{\mathrm{x}}$ & & $\mathrm{Tr}$ & \\
\hline $37 \mathrm{R}-4,35-39$ & 936.85 & & $\mathrm{Tr}$ & $\hat{x}$ & & $\hat{\mathrm{x}}$ & & & & $\hat{x}$ & & $\hat{\mathrm{x}}$ & $\hat{\mathrm{x}}$ & $\hat{\mathrm{x}}$ & & $\mathrm{x}$ & \\
\hline $38 \mathrm{R}-2,65-69$ & 943.75 & & & $\hat{x}$ & & $\hat{x}$ & & & & $\hat{x}$ & & $\hat{\mathrm{x}}$ & $\hat{\mathrm{x}}$ & $\hat{x}$ & & $\widehat{\mathrm{Tr}}$ & \\
\hline $39 \mathrm{R}-1,24-27$ & 951.54 & & $\mathrm{x}$ & $\hat{\mathrm{x}}$ & & $\hat{\mathrm{x}}$ & & & & $\hat{x}$ & & $\hat{\mathrm{x}}$ & $\hat{\mathrm{X}}$ & $\hat{x}$ & & $\mathrm{Tr}$ & \\
\hline $40 \mathrm{R}-7,0-3$ & 970.00 & & & & & $\hat{\mathrm{x}}$ & $\mathrm{K}$ ? & & $\mathrm{x}$ & $\hat{\mathrm{x}}$ & & $\hat{\mathrm{x}}$ & $\widehat{\operatorname{Tr}}$ & $\hat{x}$ & & $\mathrm{Tr}$ & \\
\hline $41 R-5,113-117$ & 977.73 & $\mathrm{x}$ & $\mathrm{x}$ & $\mathrm{x}$ & & $\hat{x}$ & $\mathrm{~K}$ ? & & & $\hat{x}$ & & $\mathrm{x}$ & $\ddot{x}$ & $\hat{x}$ & & $\mathrm{Tr}$ & \\
\hline $42 R-4,31-35$ & 985.11 & $\hat{x}$ & $\hat{x}$ & $\hat{x}$ & & & & & & $\hat{x}$ & $\mathrm{Tr}$ & $\hat{\mathrm{x}}$ & $\hat{x}$ & $\hat{x}$ & & $\mathrm{x}$ & \\
\hline $43 R-5,63-68$ & 996.33 & $\hat{x}$ & $\hat{\mathrm{x}}$ & $\hat{x}$ & & $\mathrm{x}$ & $\mathrm{K}$ ? & & & $\hat{x}$ & & $\hat{\mathrm{x}}$ & $\mathrm{x}$ & $\hat{\mathrm{x}}$ & & $\widehat{T r}$ & \\
\hline $44 \mathrm{R}-2,63-68$ & 1001.53 & $\hat{x}$ & $\hat{x}$ & $\hat{\mathrm{x}}$ & & $\hat{x}$ & $\mathrm{~K}$ ? & & & $\hat{x}$ & & $\hat{\mathrm{x}}$ & $\hat{\mathrm{x}}$ & $\hat{x}$ & & $\mathrm{Tr}$ & \\
\hline $45 R-1,38-42$ & 1009.48 & $\hat{x}$ & $\hat{x}$ & $\hat{x}$ & & & K? & & & $\hat{x}$ & & $\hat{x}$ & $\hat{x}$ & $\hat{x}$ & & & \\
\hline $46 \mathrm{R}-6,108-113$ & 1027.28 & $\hat{x}$ & $\hat{x}$ & $\hat{\mathrm{x}}$ & & & K? & & & $\hat{x}$ & & $\hat{\mathrm{x}}$ & $\hat{x}$ & $\hat{x}$ & & $\mathrm{x}$ & \\
\hline $47 R-4,25-28$ & 1032.75 & $\hat{x}$ & $\hat{x}$ & $\hat{x}$ & $\mathrm{x}$ & $\mathrm{x}$ & K? & & & $\hat{x}$ & & $\hat{x}$ & $\hat{x}$ & $\hat{\mathrm{x}}$ & & $\hat{\mathrm{Tr}}$ & \\
\hline $48 \mathrm{R}-3,58-60$ & 1041.18 & & $\hat{x}$ & $\hat{x}$ & $\hat{?}$ & $\hat{x}$ & K? & & & $\hat{x}$ & & $\hat{x}$ & $\hat{x}$ & $\hat{x}$ & & $\mathrm{Tr}$ & \\
\hline $49 \mathrm{R}-4,30-34$ & 1052.10 & $\mathrm{x}$ & $\hat{x}$ & $\hat{x}$ & & $x$ & $\mathrm{~K}$ ? & & & $\hat{x}$ & & $\hat{\mathrm{X}}$ & $\stackrel{\hat{x}}{\mathrm{x}}$ & $\stackrel{x}{x}$ & & ${ }_{\mathrm{Tr}}^{\mathrm{Ir}}$ & \\
\hline $50 \mathrm{R}-1,80-83$ & 1057.80 & & $\hat{x}$ & $\hat{x}$ & $x$ & $\mathrm{x}$ & & & & $\hat{x}$ & $\mathrm{x}$ & $\hat{x}$ & $\hat{x}$ & $\hat{x}$ & $\mathrm{x}$ & $\pi$ & \\
\hline $\begin{array}{l}50 K-1,80-83 \\
51 R-5,6-9\end{array}$ & $\begin{array}{l}1051.80 \\
1072.76\end{array}$ & $\mathrm{x}$ & $\begin{array}{l}x \\
x\end{array}$ & $\stackrel{x}{x}$ & $\begin{array}{l}x \\
\mathrm{x}\end{array}$ & $\stackrel{x}{x}$ & $\mathrm{~K}$ ? & & & $\stackrel{x}{x}$ & $\stackrel{\lambda}{\mathrm{X}}$ & $\hat{x}$ & $\stackrel{x}{x}$ & $\begin{array}{l}x \\
x\end{array}$ & $\begin{array}{l}x \\
x\end{array}$ & $\mathrm{Ir}$ & \\
\hline $\begin{array}{l}31 \mathrm{R}-1,6-9 \\
52 \mathrm{R}-93-97\end{array}$ & 1077.33 & $\stackrel{x}{x}$ & $\mathrm{x}$ & $\stackrel{x}{x}$ & $\begin{array}{l}X \\
\mathrm{x}\end{array}$ & $\stackrel{X}{\mathrm{X}}$ & $\mathrm{K}$ ? & $\frac{X}{x}$ & & $\begin{array}{l}X \\
\mathrm{x}\end{array}$ & $\begin{array}{l}x \\
\mathrm{x}\end{array}$ & $\begin{array}{l}x \\
x\end{array}$ & $\stackrel{x}{x}$ & $x$ & $x$ & ${ }_{\mathrm{Tr}}$ & \\
\hline $53 R-3,145-149$ & 1090.55 & $\hat{x}$ & & $\hat{\mathrm{x}}$ & & $\hat{\mathrm{X}}$ & $\mathrm{K}$ ? & & & $\hat{x}$ & $\hat{x}$ & $\hat{\mathrm{x}}$ & $\hat{x}$ & $\mathrm{x}$ & & $\mathrm{Tr}$ & \\
\hline $54 \mathrm{R}-3,103-107$ & 1099.73 & $\hat{x}$ & $\mathrm{x}$ & $\hat{\mathrm{x}}$ & $\mathrm{x}$ & & & $\mathrm{x}$ & & $\hat{x}$ & & $\hat{\mathrm{x}}$ & $\hat{x}$ & $\hat{\mathrm{x}}$ & & $\mathrm{Tr}$ & \\
\hline $56 \mathrm{R}-3,26-30$ & 1118.26 & & $\hat{x}$ & $\mathrm{x}$ & & $\mathrm{X}$ & & & & $\hat{x}$ & & $x$ & $\ddot{x}$ & $\mathrm{x}$ & & $\mathrm{Tr}$ & \\
\hline $57 \mathrm{R}-2,139-143$ & 1127.59 & & $\mathrm{x}$ & $\mathrm{x}$ & $\mathrm{x}$ & & & $\mathrm{x}$ & & $\mathrm{x}$ & & $\mathrm{x}$ & $\mathrm{x}$ & $\mathrm{x}$ & & $\mathrm{x}$ & \\
\hline $58 R-6,22-26$ & 1141.72 & $\mathrm{x}$ & $\mathrm{x}$ & $\mathrm{x}$ & $\mathrm{x}$ & & & $\mathrm{x}$ & & $\mathrm{x}$ & $\mathrm{Tr}$ & $\mathrm{x}$ & $\mathrm{x}$ & $\mathrm{x}$ & & $\mathrm{x}$ & \\
\hline $59 \mathrm{R}-5,74-77$ & 1150.34 & & $x$ & $\ddot{x}$ & $x$ & $\mathrm{X}$ & $\mathrm{K}$ ? & & & $x$ & $\mathrm{x}$ & $\tilde{x}$ & $\mathrm{x}$ & $\mathrm{x}$ & $\mathrm{x}$ & $\mathrm{Tr}$ & \\
\hline $60 \mathrm{R}-1,107-111$ & 1154.27 & $\mathrm{X}$ & $\ddot{x}$ & $\mathrm{x}$ & 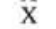 & $\mathrm{x}$ & $\mathrm{X}$ & $\mathrm{x}$ & & $\mathrm{x}$ & $\mathrm{x}$ & $\mathrm{x}$ & $\mathrm{x}$ & $\mathrm{x}$ & & $\mathrm{Tr}$ & \\
\hline $61 \mathrm{R}-2,32-36$ & 1164.72 & $\mathrm{X}$ & $\mathrm{x}$ & $\mathrm{x}$ & & & & & & $\mathrm{Tr}$ & $?$ & $\mathrm{x}$ & $\mathrm{Tr}$ & $\mathrm{x}$ & & $\mathrm{x}$ & \\
\hline $63 \mathrm{R}-3,15-19$ & 1185.35 & & & $\hat{\mathrm{x}}$ & $\mathrm{X}$ & & & & $\mathrm{x}$ & $\ddot{x}$ & & $\mathrm{x}$ & $\mathrm{x}$ & $\mathrm{x}$ & & $\mathrm{Tr}$ & \\
\hline $64 \mathrm{R}-6,14-18$ & 1199.54 & & $\mathrm{x}$ & $\hat{\mathrm{x}}$ & $\mathrm{x}$ & & & & & $\hat{x}$ & & $\hat{\mathrm{x}}$ & $\hat{\mathrm{x}}$ & $\hat{\mathrm{x}}$ & & $\mathrm{Tr}$ & \\
\hline $65 \mathrm{R}-6,101-105$ & 1210.01 & & & $\hat{\mathrm{x}}$ & & & & & & $\hat{x}$ & $\mathrm{x}$ & $x$ & $\mathrm{x}$ & $\ddot{x}$ & & $\mathrm{Tr}$ & \\
\hline $66 \mathrm{R}-6,98-101$ & 1219.58 & & $\mathrm{x}$ & $\hat{\mathrm{x}}$ & & & & & & $\hat{\mathrm{x}}$ & $\hat{\mathrm{x}}$ & $\tilde{x}$ & $\ddot{x}$ & $\hat{x}$ & & & \\
\hline $67 \mathrm{R}-1,56-60$ & 1221.36 & $\mathrm{x}$ & $\hat{x}$ & $\hat{x}$ & $\mathrm{x}$ & & & & & $\hat{x}$ & $\hat{x}$ & $\hat{\mathrm{x}}$ & $\hat{\mathrm{x}}$ & $\hat{\mathrm{x}}$ & & & \\
\hline $67 R-5,56-60$ & 1227.36 & & $\hat{\mathrm{x}}$ & $\hat{\mathrm{x}}$ & $\hat{x}$ & & & $\mathrm{x}$ & & $\hat{\mathrm{x}}$ & $\mathrm{x}$ & $\hat{x}$ & $\hat{x}$ & $\hat{\mathrm{x}}$ & & $\operatorname{Tr}$ & \\
\hline $68 \mathrm{R}-3,92-96$ & 1234.42 & & $\hat{\mathrm{x}}$ & $\hat{\mathrm{x}}$ & $\hat{x}$ & & $\mathrm{~K}$ ? & $\hat{\mathrm{X}}$ & & $\hat{x}$ & $\hat{x}$ & $\hat{x}$ & $\hat{\mathrm{x}}$ & $\hat{x}$ & & & \\
\hline $69 \mathrm{R}-2,145-148$ & 1243.15 & $\mathrm{X}$ & $\hat{x}$ & $\hat{\mathrm{x}}$ & $\hat{\mathrm{x}}$ & & & $\hat{\mathrm{X}}$ & & $\hat{x}$ & $\hat{x}$ & $\hat{\mathrm{x}}$ & $\hat{x}$ & $\hat{\mathrm{x}}$ & & & \\
\hline $70 R-2,0-4$ & 1251.30 & & $\hat{x}$ & $\hat{\mathrm{x}}$ & & $\operatorname{Tr}$ & & & & $\mathrm{x}$ & $\mathrm{x}$ & $\hat{\mathrm{x}}$ & $\widehat{x}$ & $\hat{x}$ & & & \\
\hline $71 \mathrm{R}-1,126-129$ & 1260.66 & & $\hat{x}$ & $\hat{\mathrm{X}}$ & & If & & & $\mathrm{x}$ & $\hat{x}$ & $\hat{x}$ & $\hat{\mathrm{X}}$ & $\hat{\mathrm{x}}$ & $\hat{\mathrm{X}}$ & & & \\
\hline $72 \mathrm{R}-2,0-5$ & $\begin{array}{l}1200.00 \\
1270.20\end{array}$ & & $\hat{x}$ & $\stackrel{\hat{x}}{\mathrm{x}}$ & & & & & $\lambda$ & $\hat{x}$ & $\hat{\mathrm{x}}$ & $\hat{x}$ & $\hat{\mathrm{x}}$ & $\hat{\mathrm{x}}$ & & & \\
\hline $73 \mathrm{R}-2,0-4$. & 1279.90 & & $\hat{x}$ & $\stackrel{\lambda}{x}$ & & & & & $\operatorname{Tr}$ & $\hat{\mathrm{X}}$ & $\hat{\mathrm{x}}$ & $\hat{\mathrm{X}}$ & $\hat{\mathrm{X}}$ & $\hat{\mathrm{X}}$ & & & \\
\hline $74 \mathrm{R}-3,112-116$ & 1292.12 & & $\mathrm{x}$ & $\mathrm{x}$ & & & & $\mathrm{x}$ & & $\hat{x}$ & $\hat{x}$ & $\hat{\mathrm{x}}$ & $\hat{\mathrm{x}}$ & $\hat{\mathrm{x}}$ & & $\operatorname{Tr}$ & \\
\hline $75 R-7,31-35$ & 1306.71 & & $\stackrel{x}{x}$ & $\hat{x}$ & $\mathrm{x}$ & & & $\stackrel{\lambda}{x}$ & & $\hat{x}$ & $\stackrel{\hat{x}}{\mathrm{x}}$ & $\hat{\mathrm{x}}$ & $\hat{\mathrm{X}}$ & $\hat{\mathrm{x}}$ & & ${ }_{\mathrm{Tr}}$ & \\
\hline $76 \mathrm{R}-5,77-81$ & $\begin{array}{l}1300.71 \\
1314.07\end{array}$ & & $\hat{x}$ & $\stackrel{\lambda}{\mathrm{X}}$ & $\stackrel{\hat{x}}{x}$ & & & $\stackrel{X}{\mathrm{X}}$ & & $\hat{x}$ & $\stackrel{\mathrm{X}}{\mathrm{Tr}}$ & $\hat{x}$ & $\stackrel{\hat{X}}{\mathrm{X}}$ & $\hat{x}$ & & ${ }_{\mathrm{Tr}}^{\mathrm{Ir}}$ & \\
\hline 77R-3, 15-19 & 1320.15 & & $\hat{x}$ & $\stackrel{\lambda}{x}$ & $\hat{x}$ & & & $\stackrel{X}{x}$ & & $\hat{x}$ & $\mathrm{x}$ & $\hat{\mathrm{X}}$ & $\hat{\mathrm{x}}$ & $\hat{\mathrm{X}}$ & & & \\
\hline $78 \mathrm{R}-5,31-35$ & $\begin{array}{l}1320.15 \\
133.01\end{array}$ & & ${ }_{T r}^{X}$ & $\stackrel{\lambda}{x}$ & $\mathrm{X}_{\mathrm{X}}$ & & & & & $\stackrel{x}{x}$ & $\stackrel{\hat{x}}{\mathrm{x}}$ & $\hat{x}$ & $\hat{\mathrm{x}}$ & $\hat{x}$ & & $\operatorname{Tr}$ & \\
\hline $79 R-4,141-145$ & $\begin{array}{l}1333.01 \\
1342.31\end{array}$ & & ${ }_{\mathrm{Tr}}^{\mathrm{Ir}} \mathrm{r}$ & $\stackrel{x}{x}$ & $\begin{array}{l}x \\
X\end{array}$ & & & $\mathrm{x}$ & & $\begin{array}{l}X \\
x\end{array}$ & $\stackrel{x}{\mathrm{X}}$ & $\begin{array}{l}x \\
x\end{array}$ & $\stackrel{x}{x}$ & $\begin{array}{l}X \\
x\end{array}$ & & ${ }_{\mathrm{X}}^{\mathrm{Ir}}$ & \\
\hline $\begin{array}{l}19 \mathrm{~K}-4,141-143 \\
80 \mathrm{R}-6,0-6\end{array}$ & $\begin{array}{l}1342.31 \\
1353.50\end{array}$ & & $\underset{T r}{\operatorname{Ir}}$ & $\begin{array}{l}X \\
X\end{array}$ & & $\mathrm{Tr}$ & $\mathrm{K}$ ? & $\mathrm{X}$ & & $\stackrel{x}{x}$ & $\stackrel{x}{x}$ & $\stackrel{x}{x}$ & $\stackrel{x}{x}$ & $\begin{array}{l}X \\
x\end{array}$ & & $\stackrel{x}{x}$ & \\
\hline $\begin{array}{l}80 R-6,0-0 \\
81 R-5,38-42\end{array}$ & $\begin{array}{l}1353.50 \\
1362.08\end{array}$ & $\mathrm{x}$ & Ir & $\stackrel{x}{x}$ & & $\mathrm{Tr}$ & K? & $\mathrm{x}$ & & $\begin{array}{l}X \\
x\end{array}$ & & $\stackrel{x}{x}$ & $\stackrel{x}{x}$ & $\stackrel{x}{x}$ & & $\stackrel{x}{x}$ & \\
\hline $82 \mathrm{R}-3,75-79$ & 1369.05 & & $\hat{x}$ & $\hat{\mathrm{x}}$ & & & & $\hat{x}$ & & $\hat{x}$ & $\mathrm{x}$ & $\hat{\mathrm{x}}$ & $\hat{x}$ & $\hat{\mathrm{x}}$ & & $\hat{\operatorname{Tr}}$ & \\
\hline $83 \mathrm{R}-2,21-25$ & 1376.61 & & $\hat{\mathrm{x}}$ & & $\mathrm{x}$ & & $\mathrm{x}$ & & & $\hat{x}$ & $\hat{x}$ & $\hat{\mathrm{x}}$ & $\hat{x}$ & $\hat{\mathrm{x}}$ & & & \\
\hline $84 \mathrm{R}-1,144-148$ & 1385.94 & & $\hat{\mathrm{x}}$ & & $\ddot{x}$ & $\mathrm{x}$ & & & & $\hat{x}$ & & & $\hat{x}$ & $\hat{\mathrm{x}}$ & & $\mathrm{x}$ & \\
\hline $88 R-1,112-116$ & 1395.32 & $\mathrm{X}$ & & $\mathrm{x}$ & & $\mathrm{x}$ & $\mathrm{X}$ & & & & $x$ & & & & $\mathrm{x}$ & $\hat{\mathrm{x}}$ & \\
\hline $87 R-2,79-83$ & 1425.19 & $\hat{x}$ & $\mathrm{x}$ & $\hat{\mathrm{x}}$ & & $\mathrm{x}$ & $\ddot{x}$ & & & $\mathrm{x}$ & $\hat{x}$ & & & $\mathrm{x}$ & & $\hat{x}$ & \\
\hline $89 \mathrm{R}-1,128-132$ & 1432.68 & $\mathrm{x}$ & & $\mathrm{x}$ & $\mathrm{X}$ & $?$ & $\mathrm{x}$ & & & $\hat{\mathrm{x}}$ & $\hat{x}$ & & & $\hat{\mathrm{x}}$ & $\mathrm{x}$ & $\hat{x}$ & \\
\hline $95 \mathrm{R}-1,35-38$ & 1489.35 & $\hat{x}$ & & $\mathrm{x}$ & & $\mathrm{x}$ & & & & & $\hat{x}$ & & & $\hat{x}$ & & $\hat{x}$ & \\
\hline
\end{tabular}

Notes: Key to categories: Brn cly = brown clay cement; Grn cly $=$ green clay cement; Drk red zeo $=$ zeolite with dark red (Ca) stain; Pal red zeo $=$ zeolite with pale red $(\mathrm{Ca})$ stain; Red yel zeo $=$ zeolite with red $(\mathrm{Ca})$ and yellow $(\mathrm{K})$ stains; Yel zeo $=$ zeolite with yellow $(\mathrm{K})$ stain; Uns zeo $=$ unstained zeolite $(\mathrm{n}$ ) stain); Car cem = carbonate cement; Dis bm gls = dissolution of glass (primarily $\tan /$ brown); Dis pyr = dissolution of pyroxene; Fel dis = felspar dissolution; Fel alt zeo = zeolite "replacement" (see text) of feldspar; Cly fil gls = clay-mineral alteration or "replacement" of glass; Dis yel zeo = dissolution of yellow-stained $(\mathrm{K})$ zeolite; Por = porosity; and quartz (?) cement. Key to symbols: $\mathrm{X}=$ present (left blank when none observed); $\mathrm{Tr}=$ trace; ? = uncertain identification; * = matrix-rich sample (microporous); and $\mathrm{K}$ ? = possible potassium feldspar (may be zeolite). 


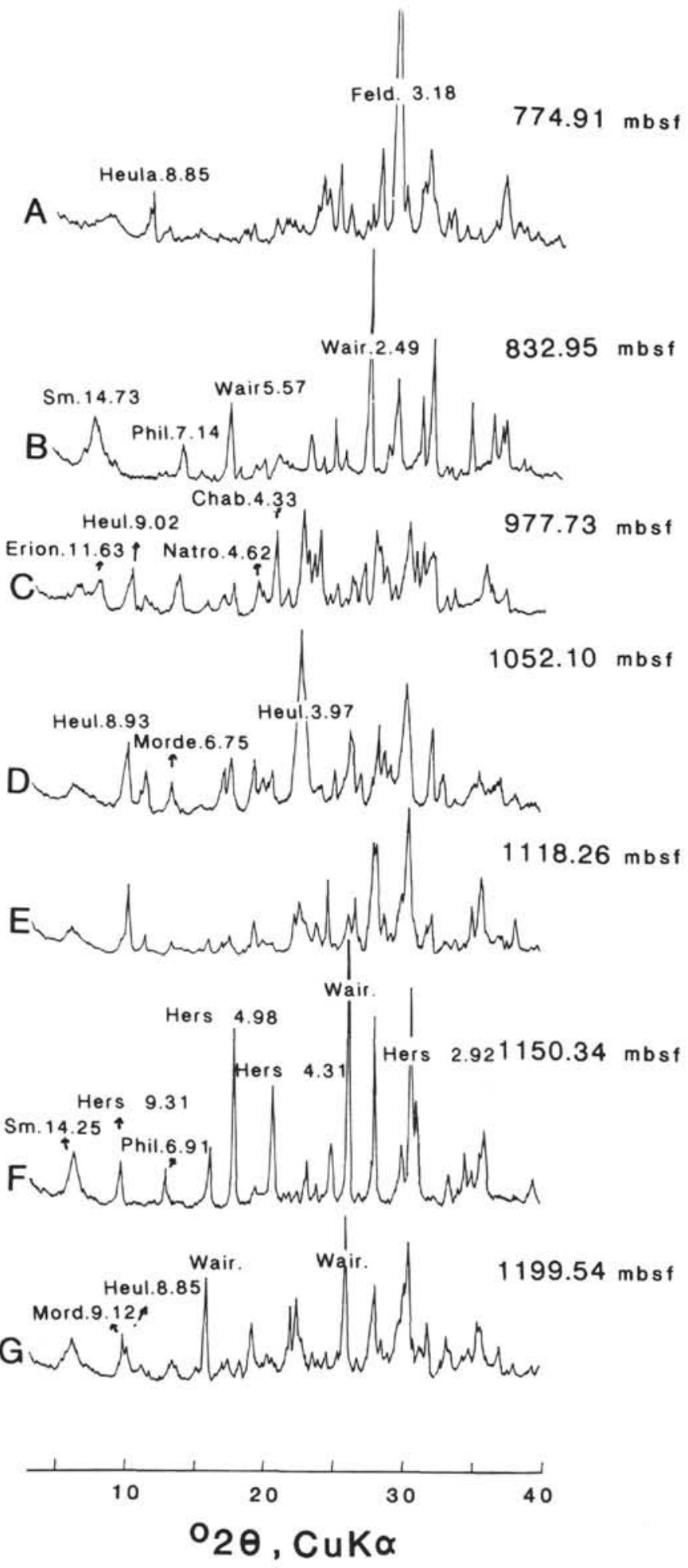

Figure 3. X-ray diffraction patterns for samples from Site 787B. Sub-bottom depths of samples are indicated on right. A. Sample 126-793B-20R-4, 91-95 cm. B. Sample 126-793B-26R-5, 125-129 cm. C. Sample 126-793B-41R-5, 113 $117 \mathrm{~cm}$. D. Sample 126-793B-49R-4, 30-34 cm. E. Sample 126-793B-56R-3, $26-30 \mathrm{~cm}$. F. Sample 126-793B-9R-5, 74-77 cm. G. Sample 126-793B-64R$6,14-18 \mathrm{~cm}$. Abbreviations are as in Figure $\mathrm{I}$ with the addition of $\mathrm{Heul}=$ heulandite, $\mathrm{Chab}=$ chabazite, Erion $=$ erionite, Natro $=$ natrolite , Morde $=$ mordenite, and Hers $=$ herschelite. minimal. This may be explained by the rapid sediment accumulation rates within the basin, which have not allowed sufficient time for diagenetic reactions to occur at depth and for the development of pore-water gradients (Taylor, Fujioka, et al., 1990). The lack of cementation in Quaternary sediments at all Leg 126 sites is not limited to the Izu-Bonin region but has been observed at all other arc-related, circum-Pacific DSDP/ODP sites (Marsaglia, 1989).

With the exception of Pliocene sandstones at Site 788, lithification in Leg 126 sandy sediments occurs in matrix-rich samples before bettersorted sands. Lithification of minimally altered volcaniclastic sandstones appears to be related to a combination of compaction and brown glass dissolution. Lithification occurs when sufficient glass has dissolved either in the sample or within nearby sediments to produce favorable conditions for smectite/zeolite precipitation. Thin smectite rims act as cohesive binders that result in sufficient induration, so that the sediments are not disaggregated by coring. Zeolite cements may also act as sediment binders, but only when they occur as grain coatings; isolated pore fillings probably do not increase the degree of induration. The best example of this process can be seen at Site 788. The progressive lithification of pumiceous sediments occurs over an interval where more lithified sections are transformed into drilling biscuits and less lithified intervals are disaggregated. Pressure welding of grains at grain contacts has also played a role in the lithification of these sediments. Compaction effects appear to be more pronounced in more deeply buried Oligocene forearc sections (as will be discussed below).

The lithification of Pliocene sands at Site 788 may be tied to its proximity to the arc axis, where geothermal gradients may be higher. Alternatively, diagenetic changes in these sediments may be relic features, produced during the deeper burial of sections now at shallower sub-bottom depths because of erosion along the rift-flank uplift. The dissolution of brown glassy fragments at Site 788 probably played a major role in the precipitation of zeolite and clay-mineral cements at this site.

The diagenetic trends outlined for forearc sites suggest an abrupt transition from fairly unaltered Miocene sediments to severely altered Oligocene sediments. Unexpectedly, however, the degree of alteration decreases with depth in the Oligocene section at Sites 787 and to some extent at nearby Site 792; this same pattern was not observed within the intensely altered/cemented section at Site 793 . These variations could be explained by regional differences in thermal history. Hydrothermal waters associated with intrusions may speed up or intensify diagenetic reactions (Boles, 1977). The section recovered at Site 793 included a shallow olivine diabase dike/sill; perhaps Oligocene volcaniclastic sandstone units acted as redistributing conduits for fluids warmed $\left(80^{\circ}-100^{\circ} \mathrm{C}\right)$ by post-Miocene intrusions in the Izu-Bonin forearc basin. When these rising hot solutions encountered the finer grained, less permeable Miocene sections, they may have spread out laterally below this permeability barrier. Such a scenario could explain the apparent higher grade of diagenesis (degree of alteration) in the site(s) most proximal to the intrusion(s) (e.g., Site 793), and, perhaps, the apparent downhole decrease in diagenetic grade at Site 787. Alternatively, an increase in geothermal gradient (up to $30 \%$ ) could have also been brought about by exothermic hydration reactions within the sediments themselves (Surdam and Boles, 1979).

If simple burial diagenesis was the major control on mineral phases present in Leg 126 sites, one would expect to see a more uniform downhole transition between zeolite facies (Surdam and Boles, 1979). The haphazard occurrence of various zeolite minerals in the Leg 126 forearc sites suggests that original sediment composition might play the most important role in controlling diagenetic reactions. Minimally altered, unconsolidated volcaniclastic sands recovered at Leg 126 sites are heterogeneous in composition and display a wide range of glass composition and mineral proportions (see Marsaglia, this volume). Within the lowermost Oligocene sections, there is also the possibility that the outer-arc high, rather than the frontal arc, was a 
sediment source (see Hiscott et al., this volume). These differences in initial sand composition could result in different zeolite assemblages under similar burial conditions (Surdam and Boles, 1979).

\section{COMPARISON OF LEG 126 DIAGENETIC TRENDS WITH THOSE FROM OTHER PHILIPPINE PLATE SITES}

Diagenetic studies of deep-marine volcaniclastic sandstones have focused on sections recovered at DSDP sites associated with remnant arcs located in marginal, backarc basins of the western Pacific Ocean (Klein and Lee, 1984; Klein et al., 1980b; Lee, 1984, 1987; Lee and Klein, 1986). In particular, these studies have concentrated on DSDP Sites 445 and 446, located in the northern Philippine Sea, on and adjacent to the Daito Ridge (Klein et al., 1980b; for location of Daito Ridge, see fig. 1 in Marsaglia, this volume). The general paragenetic sequence determined by Lee and Klein (1986) for the Eocene sections at Sites 445 and 446 is (from first to last): (1) compaction; (2) smectite and chlorite rim cements; (3) early calcite cement; (4) cristobalite, clinoptilolite, and heulandite pore-fill cements; (5) analcite cement; and (6) late calcite cement. The authigenic phases occur sporadically throughout the sand-rich sections, with only poorly defined downhole trends. In some instances, volcanic glass appears to have been directly altered to zeolites, plagioclase feldspars are altered to calcite, and these alterations increase downhole (Lee and Klein, 1986). Dissolution of feldspar, pyroxene, and volcanic glass was noted by Lee and Klein, but not documented in detail, especially with respect to the relative timing of grain dissolution.

Lee and Klein (1986) suggest that differences in the diagenetic states of Site 445 and 446 sediments are primarily controlled by initial sand composition and assumed high heat flow during backarc rifting (the lowermost sections [373-628 mbsf] cored at Site 446 consist of sediments intruded by 23 post-early Eocene basalt sills; Klein et al., 1980b).

From a downhole decrease in oxygen isotopic values, Lee (1987) calculated precipitation temperatures ranging from $23^{\circ} \mathrm{C}$ for clinoptilolite cements at $647 \mathrm{mbsf}$ to $100^{\circ} \mathrm{C}$ for analcite cements at $846 \mathrm{mbsf}$ in middle Eocene volcaniclastic sandstones. Isotopic values for latestage calcite cements indicate a narrower range of isotopic temperatures $\left(31^{\circ}-44^{\circ} \mathrm{C}\right)$, which Lee explains by post-rift thermal cooling.

Although implied by Lee's (1987) isotopic results, high heat flow during active rifting may not be a factor throughout backarc basins during their early evolution; this is shown by the lack of alteration/cementation in the Quaternary fill of the Sumisu Rift at Sites 790 and 791, and the minimal diagenesis noted by Lee and Klein (1986) at Site 453 (Mariana Basin) as compared with that observed at Sites 445 and 446. In the Pliocene sequences at Site 453, Lee and Klein (1986) describe only smectite and chlorite rim cementation and minor grain dissolution. Klein and Lee (1984) correlate sediment age with degree of alteration: the older sequences at Sites 445 and 446 are more altered than those at Site 453. This implies that time is an important factor in the alteration of volcaniclastic sediments not deposited in an active hydrothermal area.

Lee and Klein (1986) assumed that the volcanic component in the Site 445 and 446 sandstones was originally andesitic in composition. Studies of glass composition in unaltered samples from Leg 126 suggest instead that these sediments contain a heterogeneous mixture of rhyolitic to basaltic glassy fragments (see Marsaglia, and Rodolfo et al., this volume). In addition, sandstones from Sites 445 and 446 differ from those recovered at Leg 126 sites in that they contain higher proportions of limestone fragments, carbonate bioclasts, and carbonate cements.

Another major difference between Sites 445 and 446 and the Leg 126 sites is the wide age span of volcaniclastic sedimentation at the Leg 126 sites: Quaternary to Oligocene. Because of this wide age span, we have been able to document more precisely the early diagenetic reactions and lithification processes through time and under variable burial conditions.
Despite their similar intense alteration, a direct comparison between the Eocene sections at Sites 445 and 446 and the Leg 126 Oligocene forearc sections is not feasible because (1) the sediments may have been deposited in different tectonic settings, (2) the sediments are of different ages with different thermal and burial histories, (3) the original sediment compositions for these two regions were likely different, and (4) pore waters at Sites 445 and 446 were not as altered as those recovered at Leg 126 forearc sites. At Site 445, located in a small sediment pond in the Daito Ridge, $\mathrm{Ca}^{2+}$ increased downhole to a value of $45.84 \mathrm{mM}$ at $755 \mathrm{mbsf}$, whereas, at Site 446, in the Daito Basin, $\mathrm{Ca}^{2+}$ increased downhole to a value of $132.07 \mathrm{mM}$ at $595 \mathrm{mbsf}$ (Klein et al., 1980a).

\section{SUMMARY AND CONCLUSIONS}

In general, early diagenesis in Leg 126 forearc and backarc sands/sandstones is characterized by the dissolution of brown glass, the alteration of colorless glass to clay minerals, the precipitation of thin clay-mineral rim cements, and minor precipitation of clinoptilolite cements. Later, more intense diagenesis is restricted to Oligocene forearc basin sediments and includes the large-scale dissolution of feldspar and pyroxene crystals, further dissolution of vitric components, precipitation of minor carbonate cements, and pervasive zeolite cementation. The latest diagenetic events appear to be the minor dissolution of zeolite cements and the precipitation of minor carbonate and potassium feldspar (?) cements.

Although Boles and Coombs (1977) and Lee and Klein (1986) have outlined diagenetic sequences in volcaniclastic sediments, we have been able to document dissolution textures, and by looking at a range of sediment ages and burial depths, we have better quantified the time frame in which these reactions take place. Dissolution of unstable brown glass begins soon after burial ( $<1$ m.y.), lithification/cementation occurs after a few million years, and major diagenetic alteration is accomplished only after tens of millions of years. Thermal pulses brought about by intrusive events could have accelerated these reactions in older sections.

The data presented here are only preliminary findings that focus mainly on the lithification and early diagenetic changes within these sediments. Reconstructing variations in original, pre-alteration composition in sections severely altered to clay minerals and zeolites (Sites 787, 792, and 793) must be done on a textural rather than a compositional basis. Work in progress will better constrain the composition/diagenesis of the Oligocene sandstones and help decipher the complex diagenetic history of volcaniclastic sandstones within the Izu-Bonin forearc basin.

\section{ACKNOWLEDGMENTS}

This project was supported by a grant to K. M. Marsaglia from the National Science Foundation and Joint Oceanographic Institutions administered by the Texas A\&M Research Foundation. The contents of this paper do not necessarily reflect the views of the National Science Foundation, the Joint Oceanographic Institutions, Inc., or the Texas A\&M University. We thank Sharon Stonecipher and Richard Hay for thoughtful reviews.

\section{REFERENCES}

Boles, J. R, 1977. Zeolites in low-grade metamorphic rocks. In Mumpton, F. A. (Ed.), Mineralogy and Geology of Natural Zeolites. Short Course Notes, Mineral. Soc. Am., 4:103-135.

Boles, J. R., and Coombs, D. S., 1977. Zeolite facies alteration of sandstones in the Southland Syncline, New Zealand. Am. J. Sci., 277:982-1012.

Coombs, D. S., Ellis, A. J., Fyfe, W. S., and Taylor, A. M., 1959. The zeolite facies, with comments on the interpretation of hydrothermal syntheses. Geochim. Cosmochim. Acta, 17:53-107. 
Egeberg, P. K., and the Leg 126 Shipboard Scientific Party, 1990. Unusual composition of pore waters found in the Izu-Bonin fore-arc sedimentary basin. Nature, 344(6263):215-218.

Fisher, R. V., and Schmincke, H.-U., 1984. Pyroclastic Rocks: New York (Springer-Verlag)

Friedman, G. M., 1971. Staining. In Carver, R. E. (Ed.), Procedures in Sedimentary Petrology: New York (Wiley-Interscience), 511-530.

Hay, R. L., 1977. Geology of zeolites in sedimentary rocks. In Mumpton, F. A. (Ed.), Mineralogy and Geology of Natural Zeolites. Short Course Notes, Mineral. Soc. Am., 4:54-63.

Kastner, M., 1981. Authigenic silicates in deep-sea sediments: formation and diagenesis, In Emiliani, C. (Ed.), The Sea (Vol. 7): The Oceanic Lithosphere: New York (Wiley-Interscience), 915-980.

Klein, G. deV., Kobayashi, K., et al., 1980a. Init. Repts. DSDP, 58: Washington (U.S. Govt. Printing Office).

Klein, G. deV., and Lee, Y. I., 1984. A preliminary assessment of geodynamic controls on depositional systems and sandstone diagenesis in back-arc basins, western Pacific Ocean. Tectonophysics, 102:119-152.

Klein, G. deV., McConville, R. L., Harris, J. M., and Steffensen, C. K., 1980 b. Petrology and diagenesis of sandstones, Deep Sea Drilling Project Site 445, Daito Ridge. In Klein, G. deV., Kobayashi, K., et al., Init. Repts. DSDP, 58: Washington (U.S. Govt. Printing Office), 609-616.

Lee, Y. I., 1984. Petrology and diagenesis of medium-grained clastic sediments in the back-arc basins of the western Pacific Ocean [Ph.D. dissert.]. Univ. Illinois, Urbana.

, 1987. Isotopic aspects of thermal and burial diagenesis of sandstones at DSDP Site 445, Daito Ridge, northwest Pacific Ocean. Chem. Geol., 65:95-102.

Lee, Y. I., and Klein, G. deV., 1986. Diagenesis of sandstones in the back-arc basins of the western Pacific Ocean. Sedimentology, 33:651-675.

Marsaglia, K. M., 1989. Petrography, provenance, and diagenesis of arc-related sands recovered by the Deep Sea Drilling Project on circum-Pacific, Mediterranean and Caribbean legs [Ph.D. dissert.]. Univ. California, Los Angeles.

Seki, Y., Onuki, H., Okumura, K., and Takashima, I., 1969. Zeolite distribution in the Katayama geothermal area, Onikobe, Japan. Jpn. J. Geol. Geog., 40:63-79.

Shiraki, R., and liyama, T., 1990. Na-K ion exchange reaction between rhyolitic glass and $(\mathrm{Na}, \mathrm{K}) \mathrm{Cl}$ aqueous solution under hydrothermal conditions. Geochim. Cosmochim. Acta, 54:2923-2931.
Surdam, R. C., and Boles, J. R, 1979. Diagenesis of volcanic sandstones. In Scholle, P. A., and Schluger, P. R. (Eds.), Aspects of Diagenesis. Spec. Publ., Soc. Econ. Paleontol. Mineral., 26:227-242.

Taylor, B., Fujioka, K., et al., 1990. Proc. ODP, Init. Repts., 126: College Station, TX (Ocean Drilling Program).

Date of initial receipt: 2 January 1991

Date of acceptance: 17 May 1991

Ms 126B-123

\section{APPENDIX}

The staining technique used is modified from techniques outlined in Friedman (1971). In this method, the thin section is immersed in concentrated hydrofluoric acid for $3 \mathrm{~s}$, after which the thin section is quickly dipped once in a large beaker of distilled water, and then immersed for $25 \mathrm{~s}$ in a supersaturated solution of sodium cobaltinitrite. After this, the thin section is gently washed by dipping it twice into a tub of distilled water; it is then gently dried with low-velocity compressed air (critical factor) and left overnight. The following day, the thin section is first immersed in a supersaturated solution of barium chloride for $10 \mathrm{~s}$, quickly dipped once in a large beaker of distilled water, and then placed for $30 \mathrm{~s}$ in a supersaturated solution of amaranth. After two slow dips in a tub of distilled water, the thin section is gently dried with low-velocity compressed air and left overnight before application of a coverslip. In each step, air velocity during drying is critical: air with too high a velocity will remove the gel/stain from grain surfaces. Note that different containers of distilled water are used during the various steps and that those used to rinse off staining chemicals need to be changed often. This method requires a very good hood and acid-resistant protective gloves, apron, and goggles (see hazards outlined in Friedman, 1971). Acid and acid rinse water should be neutralized under a hood.

When observed with an optical microscope under plane-polarized light, the calcium content of feldspar is reflected in the intensity of the stain. Fresh plagioclase takes on a red stain whereas more albitic varieties take on a lighter pink stain. The potassium feldspar stain is light yellow and is concentrated along cleavage traces. The glassy groundmass of volcanic rock fragments, gypsum, and authigenic zeolites are also stained according to their calcium and potassium content using this method. 


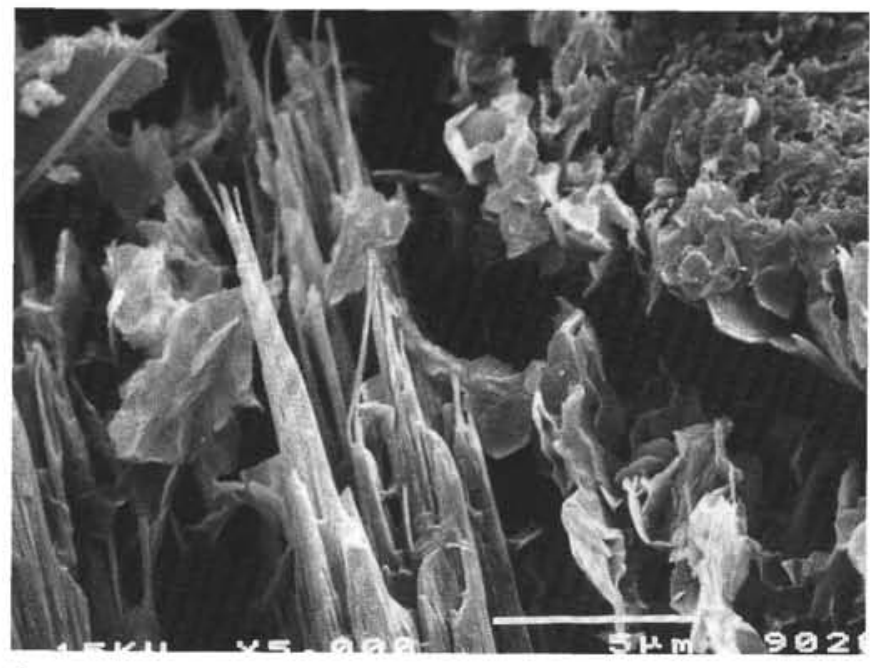

1

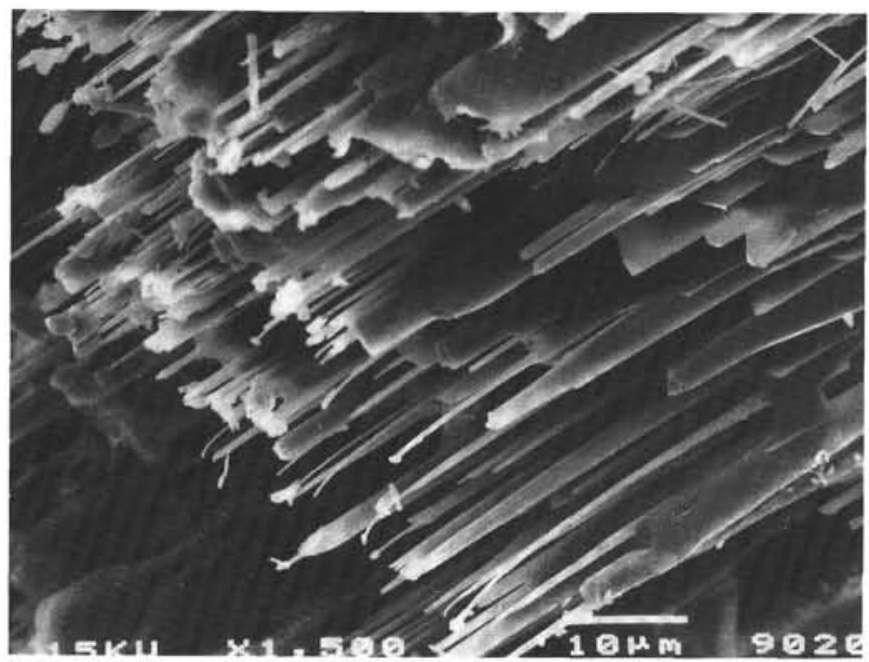

2

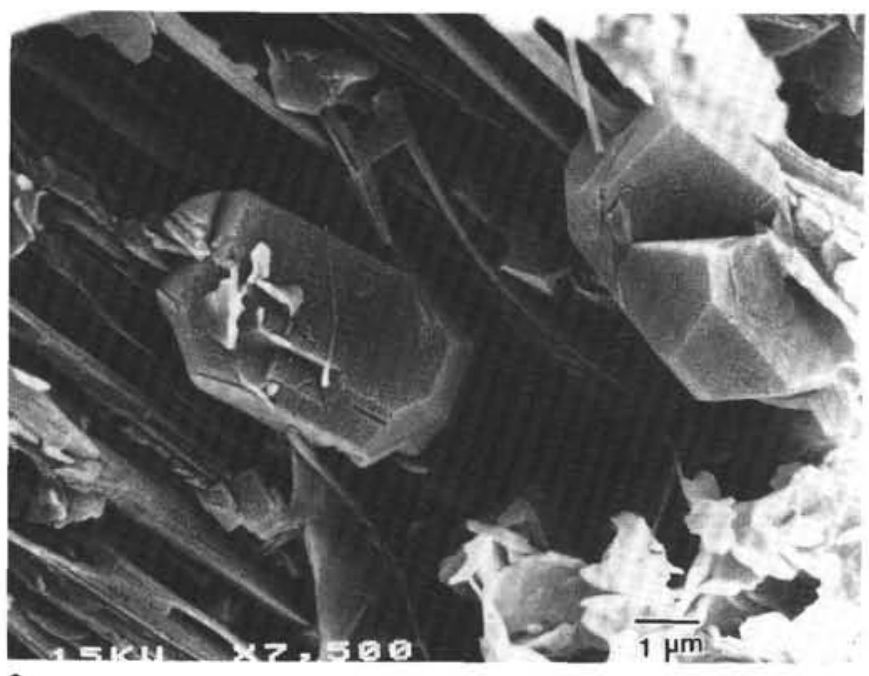

3

Plate 1. Scanning electron microscope photographs. 1. Needle or thin fibrous erionite with filmy smectite (Sample 126-793B-41R-5, 113-117 cm). 2. Parallel-connected, needle- or lath-shaped crystals of mordenite with C elongation (Sample 126-793B-59R-5, 74-77 cm). 3. Heulandite crystals, some are (010) lamellae, other larger crystals are quasi-orthogonal (Sample 126-793B-59R-5, 74-77 cm). Irregular surfaces on these crystals suggest the presence of secondary minerals. 


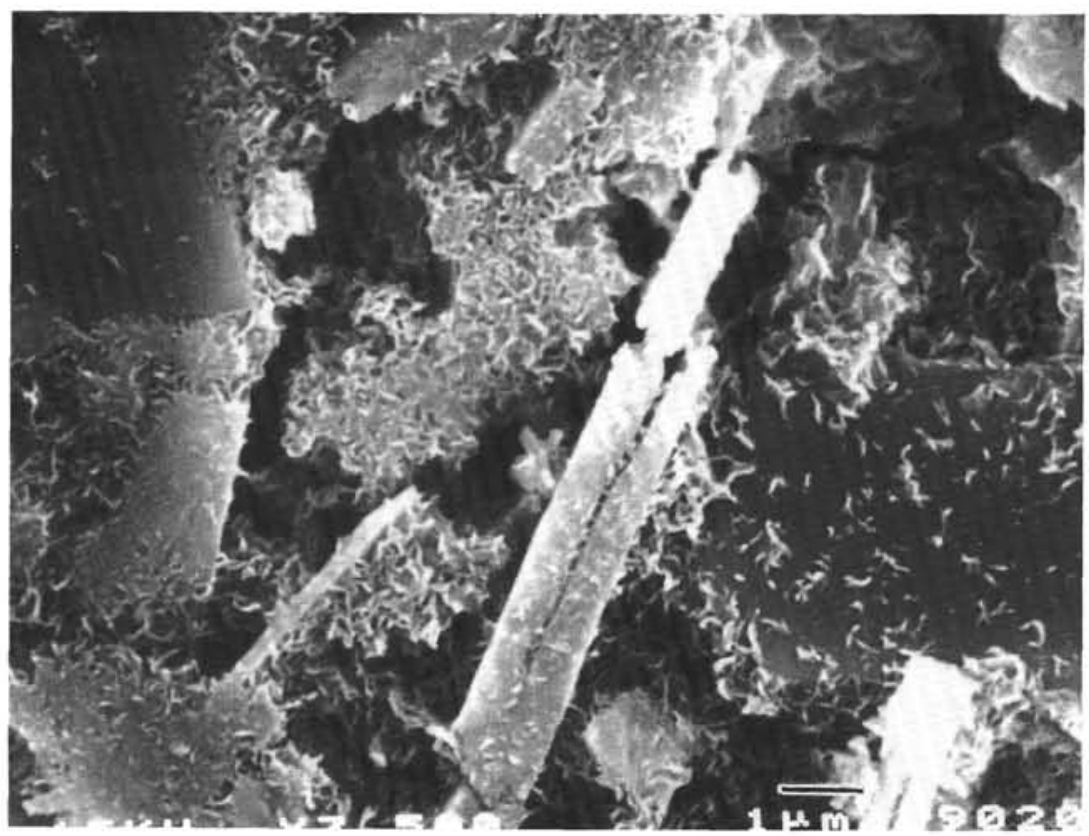

1

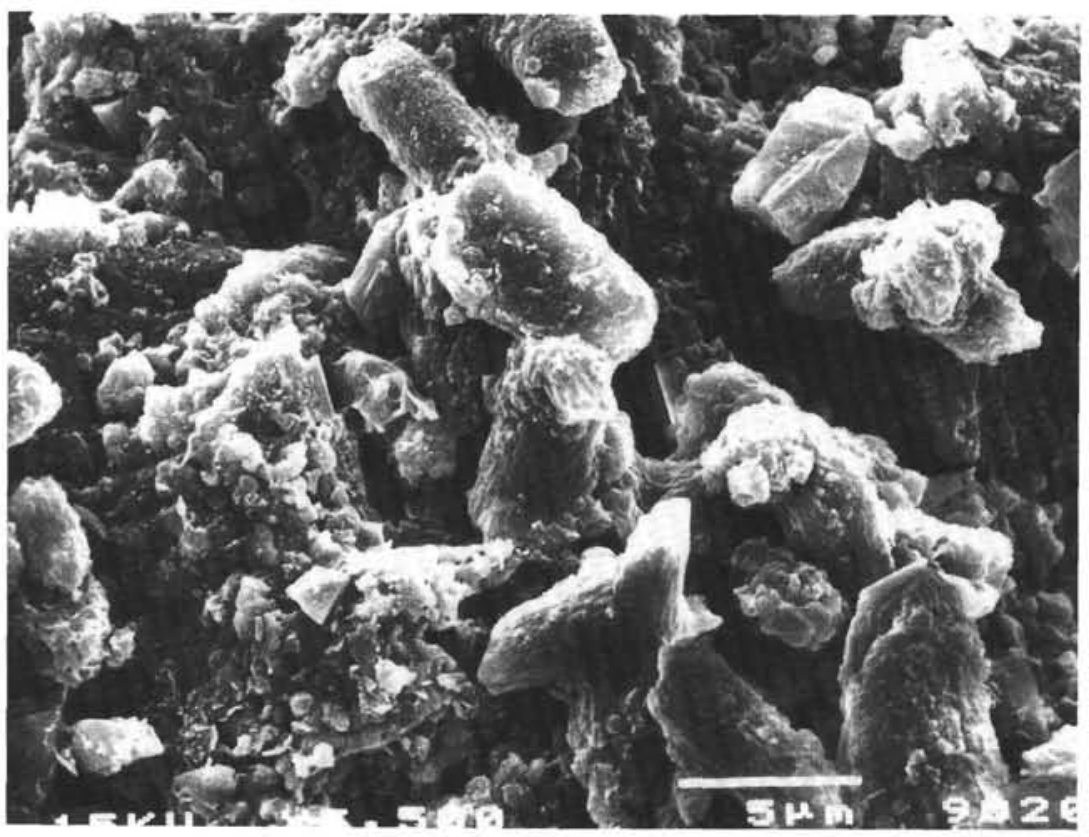

2

Plate 2. Scanning electron microscope photographs. 1. Irregular film of smectite covering surfaces of zeolite (phillipsite and wairakite), feldspar and calcite crystals (Sample 126-787B-15R-1, 108-111 cm). 2. Defaced surface of wairakite and feldspar covered by small particles of secondary minerals with smectite (Sample 126-792E-47R-2, 88-93 cm). 

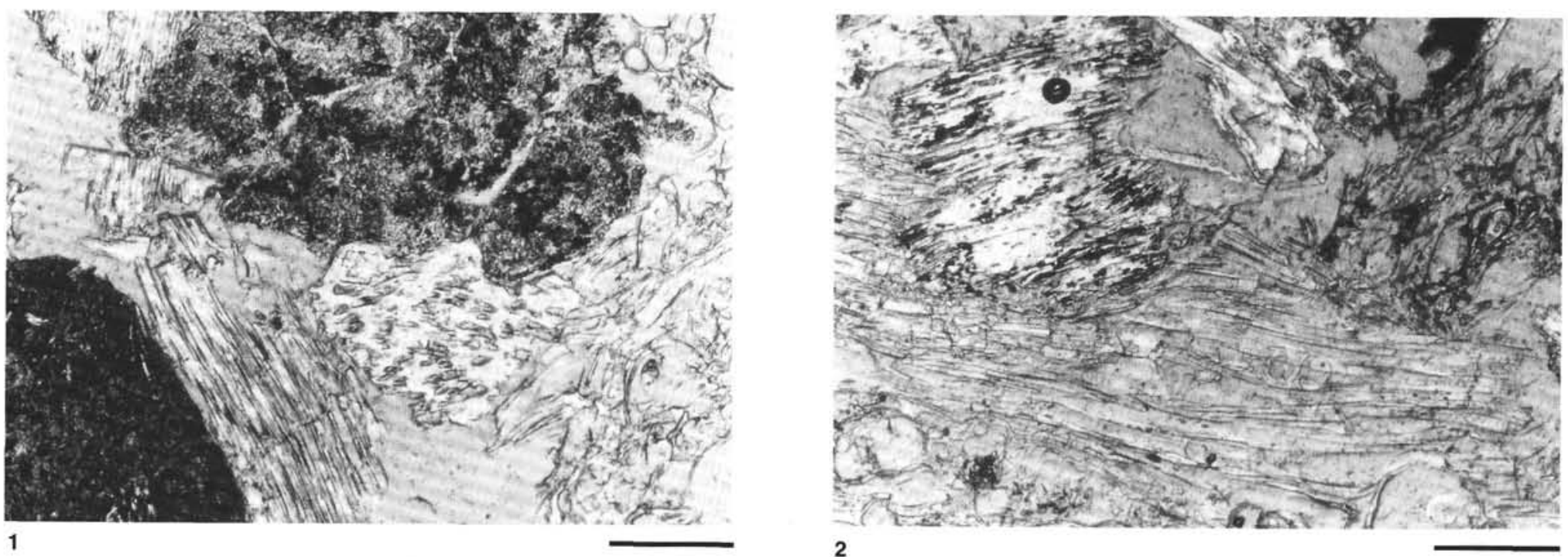
2
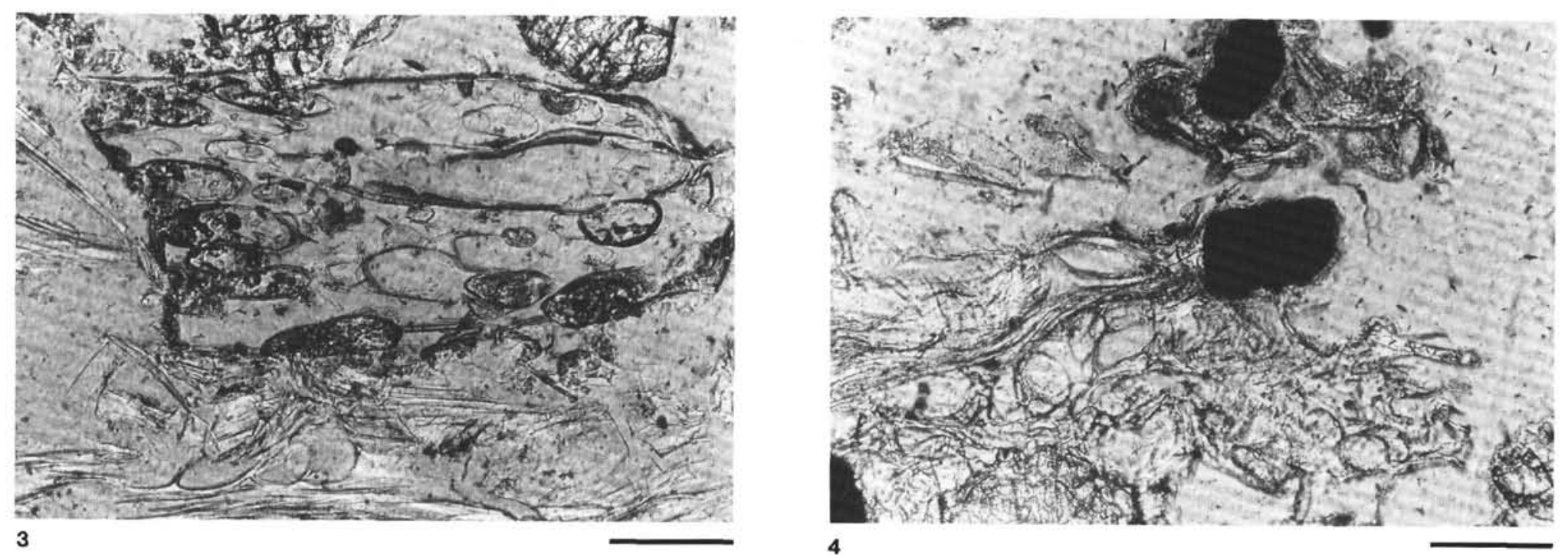

Plate 3. Photomicrographs, all plane-polarized light. 1. Compactional features in Sample $126-788 \mathrm{C}-20 \mathrm{H}-2,40-42 \mathrm{~cm}$. Altered volcanic grain (top) is pressure-welded to vesicular white glass fragment below. The pumice fragment located between two dark volcanic grains is fractured. Porosity is light gray; note the mostly vesicular, highly porous pumice fragment on left. Stained/etched portion of slide. The scale bar equals $0.2 \mathrm{~mm}$. 2. Compactional features in Sample $126-788 \mathrm{C}-20 \mathrm{H}-2,40-42 \mathrm{~cm}$. Interpenetration of less vesicular glass grains (white and dark gray: top) into highly vesicular pumice friog of slide. The scale bar is light gray. Dark gray glass grain is partially dissolved. Stained/etched portion of slide. The scale bar equals $0.2 \mathrm{~mm}$. 3. Dissolved microlitic/vesicular brown(?) glass in Sample $126-788 \mathrm{C}-19 \mathrm{H}-1,69-71 \mathrm{~cm}$. Interparticle and intraparticle porosity is light gray. Grain and vesicles outlined by a thin, discontinuous clay mineral (smectite?) rim. Only isolated microlites are left "floating" in a secondary pore. Stained/etched portion of slide. The scale bar equals $0.1 \mathrm{~mm}$. 4. Partial alteration of colorless pumice adjacent to opaque microphenocrysts (Sample 126-788C-19H-1, 69-71 cm). Clay-mineral alteration products are dark gray and porosity is light gray. Stained/etched portion of slide. The scale bar equals $0.1 \mathrm{~mm}$. 

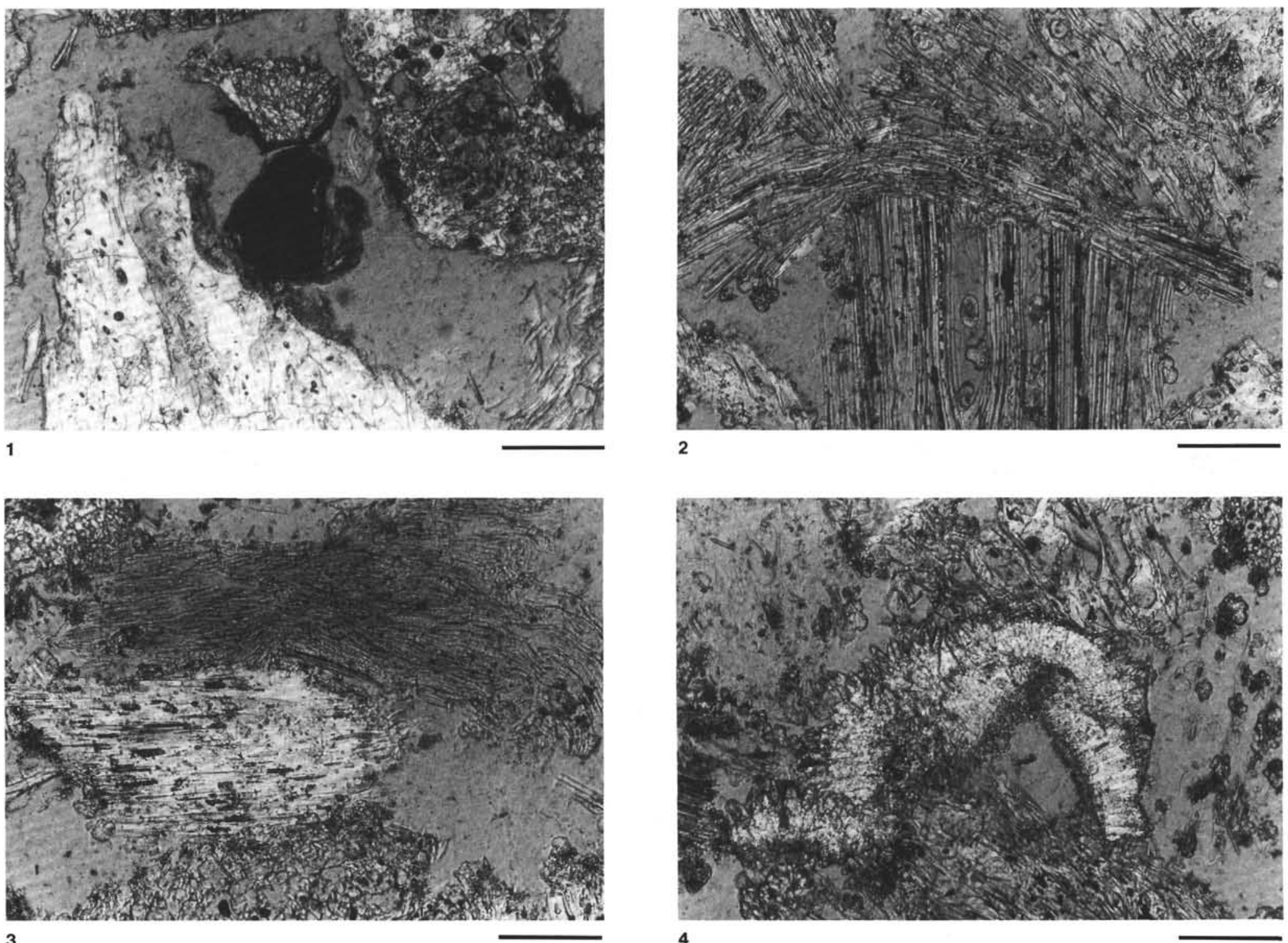

Plate 4. Photomicrographs, all plane-polarized light, and scale bars equal $0.1 \mathrm{~mm}$. 1. Thick clay-mineral rim cements developed only adjacent to opaque grain; Sample $126-788 \mathrm{C}-19 \mathrm{H}-1,69-71 \mathrm{~cm}$. Clay-mineral rim cements are dark gray and porosity is light gray. Stained/etched portion of slide. 2. Compactional features and incipient cementation in Sample 126-788D-11R-1, 17-20 cm. Pumice fragment in center is bent by compaction. Incipient zeolite (phillipsite?) cementation occurs as small globular clusters of submicroscopic crystals within interparticle pores (light gray) and intraparticle vesicles. Globules appear white when protected from etching/staining by a thin film of epoxy, and dark gray when stained. 3. Pumice fragment completely altered to clay minerals (dark gray) adjacent to unaltered grain of vesicular glass (white) (Sample 126-788D-11R-1, 17-20 cm). Porosity is light gray. Stained/etched portion of slide. 4. Crushed calcareous foraminifer test (white) in Sample 126-788D-IIR-1, 17-20 cm. Unaltered colorless pumice grains above and below, pore spaces to left and right result from the dissolution of brown glass. Outlines of vesicles and microlites are barely visible in secondary pores (light gray). Small dark/ight clusters are variably stained incipient zeolite. Stained/etched portion of slide. 

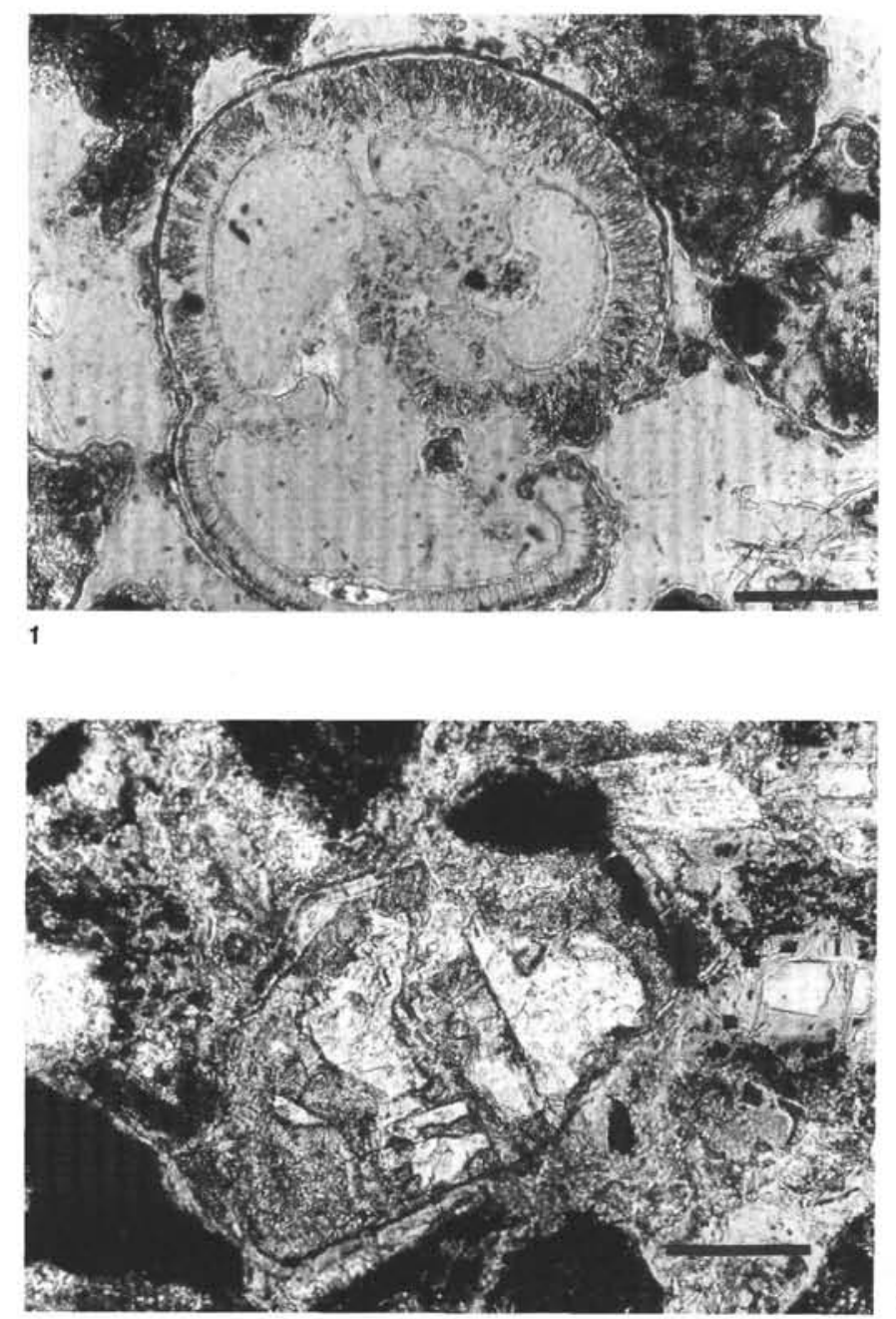

2

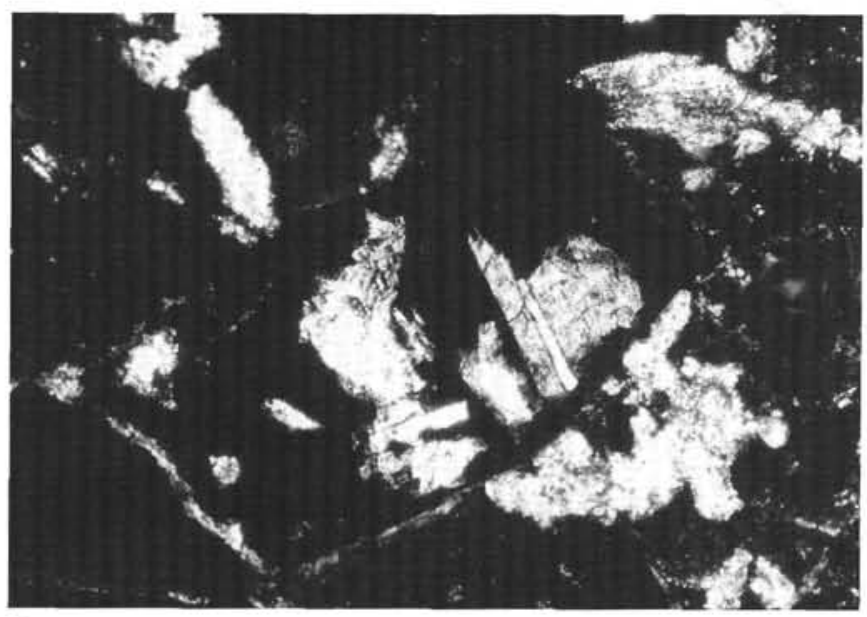

Plate 5. Photomicrographs, scale bars equal $0.1 \mathrm{~mm}$. 1. Partially dissolved foraminifer test in Sample 126-788D-5R-1,42-45 cm. Carbonate skeletal remnants are dark gray, secondary porosity is light gray. Grains are coated with a thin rim of clay mineral cement (dark gray). Speckled light/dark grain is partially dissolved brown glass. Stained/etched portion of slide. Plane-polarized light. 2. Partially dissolved plagioclase feldspar grain in Sample 126-793-19R-4, 47-51 cm. Intraparticle porosity has been filled by red-stained zeolite; interparticle cements include carbonate, mixed-stained zeolites and birefringent chlorite cements. Stained/etched portion of slide. Plane-polarized light. 3. Same as in Figure 2 with crossed nicols. 